Review Article

\title{
Probiotics as a New Regulator for Bone Health: A Systematic Review and Meta-Analysis
}

\author{
Hanieh Malmir, ${ }^{1,2}$ Hanieh-Sadat Ejtahed ${ }^{1},{ }^{1,3}$ Ahmad-Reza Soroush, ${ }^{1}$ \\ Amir Mohammad Mortazavian, ${ }^{4}$ Noushin Fahimfar, ${ }^{5}$ Afshin Ostovar, ${ }^{5}$ \\ Ahmad Esmaillzadeh, ${ }^{1,2}$ Bagher Larijani, ${ }^{3}$ and Shirin Hasani-Ranjbar $\mathbb{D}^{1,3}$ \\ ${ }^{1}$ Obesity and Eating Habits Research Center, Endocrinology and Metabolism Clinical Sciences Institute, \\ Tehran University of Medical Sciences, Tehran, Iran \\ ${ }^{2}$ Department of Community Nutrition, School of Nutritional Sciences and Dietetics, Tehran University of Medical Sciences, \\ Tehran, Iran \\ ${ }^{3}$ Endocrinology and Metabolism Research Center, Endocrinology and Metabolism Clinical Sciences Institute, \\ Tehran University of Medical Sciences, Tehran, Iran \\ ${ }^{4}$ Department of Food Science and Technology, Faculty of Nutrition Sciences and Food Technology, \\ Shahid Beheshti University of Medical Sciences, Tehran, Iran \\ ${ }^{5}$ Osteoporosis Research Center, Endocrinology and Metabolism Clinical Sciences Institute, Tehran University of Medical Sciences, \\ Tehran, Iran
}

Correspondence should be addressed to Hanieh-Sadat Ejtahed; haniejtahed@yahoo.com and Shirin Hasani-Ranjbar; sh_hasani@tums.ac.ir

Received 29 May 2021; Revised 15 July 2021; Accepted 21 July 2021; Published 3 August 2021

Academic Editor: Vahidreza Ostadmohammadi

Copyright (c) 2021 Hanieh Malmir et al. This is an open access article distributed under the Creative Commons Attribution License, which permits unrestricted use, distribution, and reproduction in any medium, provided the original work is properly cited.

\begin{abstract}
Despite the proposed role of the gut microbiota-bone axis, findings on the association between probiotic consumption and bone health are conflicting. This systematic review aimed to assess the effect of probiotic consumption on bone health parameters. A systematic literature search of relevant reports published in PubMed/Medline, Web of Science, SCOPUS, EMBASE, and Google scholar before December 2020 was conducted. All clinical trials or experimental studies, which examined the relationship between probiotic consumption and bone health parameters, were included. No limitation was applied during the search. After screening articles based on inclusion criteria, 44 studies remained. In clinical trials, probiotic consumption affects bone health parameters such as serum calcium levels (3.82; 95\% CI: 1.05, $6.59 \mathrm{mmol} / \mathrm{l})$, urinary calcium levels $(4.85 ; 95 \% \mathrm{CI}: 1.16,8.53 \mathrm{mmol} / \mathrm{l})$, and parathyroid hormone (PTH) levels $(-5.53 ; 95 \% \mathrm{CI}:-9.83,-0.86 \mathrm{ng} / \mathrm{l})$. In most studies, Lactobacillus species such as L. helveticus, L. reuteri, and $L$. casei were consumed and women aged 50 years or older were assessed. Spinal and total hip bone mineral density (BMD) was not affected significantly by probiotic consumption. In 37 animal experiments, probiotic or symbiotic feeding mostly had effects on bone health parameters. Some strains of Bifidobacterium and Lactobacillus including L. reuteri, L. casei, L. paracasei, L. bulgaricus, and L. acidophilus have indicated beneficial effects on bone health parameters. In conclusion, this systematic review and meta-analysis indicate that probiotic supplementation might improve bone health. Further studies are needed to decide on the best probiotic species and appropriate dosages.
\end{abstract}

\section{Introduction}

Bone health is critically important to the overall health and quality of life and depends on the balance of bone resorption and bone formation [1]. Low bone mineral density (BMD) value is an indicator of osteoporosis or fracture and one of the major public health problems [2]. Significant disability, increased dependency, reduced quality of life, and increased economic burden to the health care system are the most consequences of reduced BMD [3-5]. BMD is affected by 
various factors, including gender, age, family history, fracture history, alcohol consumption, tobacco smoking, taking some medicines, bone diseases, and lack of physical activity [6-11]. Moreover, previous studies indicated that hormone status, immune cells, and the gastrointestinal system can also regulate bone balances and health [12]. The gastrointestinal tract has a key role in the absorption of calcium, phosphorous, and magnesium as contributors to bone mineralization and produces endocrine factors that signal to bone cells, such as Incretin and serotonin. Furthermore, the gut microbiota has been proposed as a regulator of bone health [13].

The human gut microbiota comprises over 1000 distinct microbial species [14]. According to Codex Alimentarius, probiotics are live microorganisms that provide health benefits, generally by improving or restoring the gut microbial flora [15]. Although, they are recommended to be defined as the viable or unviable microbial cell (vegetative or spore; intact or ruptured) that is potentially healthful to the host [16]. Disturbed gut microbiota composition contributes to many human chronic diseases, such as obesity, metabolic diseases, malnutrition, neurological disorders, cardiovascular disease, and cancer [17]. Also, bone mass and bone quality are affected by gut microbiota [18]. Previous studies have indicated the relation between bone health and probiotics. For example, in Takimoto et al. study, oral probiotic supplementation stimulates the diversity of gut microbiota as well as bone growth and density [19]. Although most of the previous publications indicated beneficial effects of probiotics on bone health [19-21], some others had not found any association between probiotic consumption and bone health status [22, 23]. In Sergeev et al.'s study, three-month supplementation by a probiotic capsule had no effects on the bone mineral content in overweight and obese adults [22]. Despite these controversies, no publication summarized findings from previous publications in this field. Although bone health and diseases have a significant role on affected individuals, they also affect the population [24]. Osteoporosis has increased economic burden to health care system, as well as loss of income to the employee, loss of productivity to the employer, costs to a country's social welfare system including unemployment and disability pay, health insurance payments, and rises in insurance premiums [25]. Due to the deep effects of osteoporosis and fractures on society and increasing prevalence of osteoporosis [26], it seems that preventing studies are more important. This study, therefore, aimed to comprehensively review previous animal studies and clinical trials about the effect of probiotic consumption on bone health status.

\section{Materials and Methods}

This systematic review was performed according to the Preferred Reporting Items for Systematic Reviews and MetaAnalysis (PRISMA) statement with the aim of assessing the relation between probiotic consumption and bone health parameters and has been recorded in PROSPERO.
2.1. Search Strategy. Previous publications on the effect of probiotic consumption and bone health status were selected through searching in PubMed/Medline, ISI Web of Science, SCOPUS, Cochrane, and EMBASE up to December 2020. The following key words were used in this search: probiotics OR synbiotic OR Lactobacillus OR Bifidobacterium for the intake of probiotics, and osteoporosis OR fracture OR "bone mineral density" OR BMD OR "bone mineral content" OR "alkaline phosphatase" OR osteocalcin OR "procollagen type $1 \mathrm{~N}$-terminal propeptide" OR hydroxyproline OR "NF- $\mathrm{KB}$ ligand" for bone health status (Supplemental Table 1). In PubMed, keywords were searched through [tiab] and [MeSH] tags. No limitation was applied during the search. The reference lists of retrieved papers were also examined to avoid missing any published data. Finally, articles in the English language were included.

2.2. Inclusion and Exclusion Criteria. Two investigators independently selected the articles through the mentioned search strategy. Publications that fulfilled the following criteria were eligible for inclusion: (1) all studies with clinical trial design or experimental design (animal studies); (2) studies that examined the relationship between probiotic consumption and bone health status parameters; and (3) those that reported quantity findings for probiotic consumption and bone health parameters. We excluded letters, comments, reviews, meta-analyses, ecological, and in vitro studies as well as duplicate studies. Inclusion criteria based on PICOS include the following: Population, adults (for human studies) and other animals for experimental studies; Intervention/Exposure, probiotic consumption; Comparison, consumption and nonconsumption of probiotics; Outcome: bone health parameters; Study design: clinical trial design or experimental design (animal studies).

2.3. Data Extraction. For each eligible study, the following information was extracted: first author, year of publication, study design, country, age range, gender, sample size (number of participants in each group), type of intervention, duration of intervention, the dose of probiotic intake in the intervention group, characteristics of the control group, outcome variables, the mean and standard deviation of bone health parameters in the intervention and control groups, and quality score.

2.4. Quality Assessment of Studies. The risk of bias for the included studies was evaluated using the Cochrane quality assessment tool for RCTs. Two independent investigators assessed the quality of studies using the following seven criteria: (i) random sequence generation, (ii) allocation concealment, (iii) blinding of participants and personnel, (iv) blinding of outcome assessment, (v) incomplete outcome data, (vi) selective reporting, and (vii) other probable sources of biases. To evaluate the quality of studies, each study was allocated a label (yes, no, or unclear) indicating that it was judged as low risk, high risk, or unknown risk of bias, respectively [27]. 
All steps of the methods were performed by two investigators independently including searching, article screening, and data extracting, and checking the quality of articles. Disagreements between the two investigators were resolved by discussion and consensus.

2.5. Statistical Analysis. Mean differences \pm SDs of measures such as chemical bone health parameters and BMD, comparing probiotic consumption to control, were used to calculate the overall effect sizes. When mean differences \pm SDs were not reported, we calculated them by considering changes in each parameter throughout the study. In addition, these parameters were reported in different units across the studies. We converted them to the same units. The overall effect size was calculated by using a random effects model, which takes between-study variation into account. Cochran's Q test and I2 statistic were used to assess between-study heterogeneity. Sensitivity analysis was used to explore the extent to which inferences might depend on a particular study or group of studies. Publication bias was examined by visual inspection of funnel plots and the application of Egger's and Begg's tests. We used kappa statics to assess the consonant between investigators. All statistical analyses were conducted by using STATA version 14.2 (StataCorp). $P$ values $<0.05$ were considered significant.

\section{Results}

In total, 1123 articles were found in our initial search. After exclusion of duplicate studies and screening nonrelated articles based on title and abstract, 75 articles were remained. We further excluded 31 papers because of the following reasons: (1) those that examined the effect of probiotic consumption on gut microbiota without considering the effects of probiotics on bone health status or assessed the relationship between gut microbiota and bone health parameters without intervention $(n=16)$; (2) publications in which no effect sizes were reported $(n=3)$; and (3) those that had observational design (cohort, case-control, or crosssectional design) $(n=12)$. After these exclusions, 44 papers remained for the current systematic review (Figure 1). Two investigators independently selected the articles through the mentioned search strategy and they had high agreement (0.90). The disagreement between the two investigators was resolved by the opinion of the third one.

3.1. Animal Studies. Characteristics of 37 animal studies on the effects of probiotics on bone parameters are presented in Table 1. These investigations are published between 2004 and 2020. Most of them were performed on rats except for four studies performed on chicks and hens [28-31]. Target species of rats were Sprague-Dawley in 10 studies [32-41], C57BL/6J mice in 9 studies [42-50], Wistar rat in 5 studies [51-55], and BALB/c mice in 5 studies [56-59]. The other studies used senescence-accelerated mouse (SAMP) [60, 61], virgin fisher rat [62], and ND4 Swiss Webster retired breeder mice [63]. Out of 37 studies, 17 publications were performed on male $[29,33,35,38,41,45,46,48,49,52-55,60$,
$61,63,64], 17$ on female $[30,32,34,36,37,39,40,42$, $44,50,51,56,57,59,62,65]$, and 3 on both gender $[28,31,43]$. In fourteen investigations, female rats had ovariectomy surgery that induced osteoporosis $[36,37,39,40,42,44,47,51,56,57,59,62,66]$, and in two studies, diabetic rats were included $[38,45]$. Animals were fed by $L$. reuteri $[30,31,40,43,45,46,56,57,64]$, L. casei [34, 38-40, 51, 63], L. paracasei [42, 44, 54, 65], L. plantarum [42, 47, 63, 65], L. acidophilus [40, 55, 59, 67], B. bifidum $[40,53,55,63]$, B. longum $[37,47,52,53]$, B. subtilis $[28,29,55]$, L. helveticus $[32,37,41]$, L. bulgaricus [33, 35, 63], Entrococcos faecium [30, 31, 55], and L. rhamnosus $[48,49,64]$. Other studies used $B$. breve [53, 63], B. animalis [30, 31], Streptococcus thermophilus [33, 35], Pediococcus acidilactici [30, 31], Escherichia coli [49, 64], Lactococcus lactis [60, 61], Bacillus licheniformis [28], Clostridium butyrium [29], Bacillus coagulans [40], and Pasteurized Akkermansia muciniphila [50]. The dosage and complete name and species of probiotics were reported in Table 2. The sample size varied from 1 [61] to 120 [30] in each group. The duration of intervention was between 9 days $[33,35]$ and 11 months [61]. Although probiotic feeding had increased calcium [39], phosphorus [42, 48], 25-OH-D $[40,50]$, PTH [33], osteocalcin (OC) $[33,36,44,50,51]$, and alkaline phosphatase (ALP) [40] levels in some investigations, reduced levels of ALP [39], acid phosphatase (ACP) [65], urinary calcium [41], and phosphorus [48] were observed in others. These different findings might be due to different age, sex, estrogen status, duration of intervention, and sample sizes. In terms of BMD, increased BMD in different sites were reported in previous publications, total $[34,35,44,51,55]$, tibia $[12,30-32,35,54,59]$, femur $[30,31,49,59]$, and calcaneus [35]. An increase in BMC was also reported in five investigations [30, 31, 40, 43, 57]. Trabecular thickness $[34,39,43-45,50,51,56,59]$, bone volume $[32,34,36,38,39,42-44,49,50,57,59,65]$, tibia length [29], femur weight [38, 65], and bone phosphorus $[28,52]$ and bone calcium $[52,53]$ were also affected by probiotic feeding in animals. In eight publications, probiotic feeding showed no effects on bone parameters $[37,46,47,60-64]$.

3.2. Clinical Trials. Characteristics of seven clinical trials regarding the effects of probiotic consumption on bone health status presented in Table 2. These studies are published between 2004 and 2020. Three of the publications were performed in European countries [20, 21, 68], two in Asian countries $[19,23]$, and two in the USA and Canada [22, 69]. All studies had randomized study design except for two of them $[22,68]$. Out of seven included clinical trials, three studies were conducted on healthy postmenopausal women $[19,21,68]$, two on postmenopausal women with osteopenia $[20,23]$, one in hypercholesterolemic adults [69], and one in overweight and obese adults [22]. Sample sizes were varied from 10 to 66 in the intervention group and 10 to 61 in the control group. The dosage and complete name and species of probiotics are reported in Table 2. Supplements contained $1.5^{*} 10^{8}$ to $5^{*}$ 


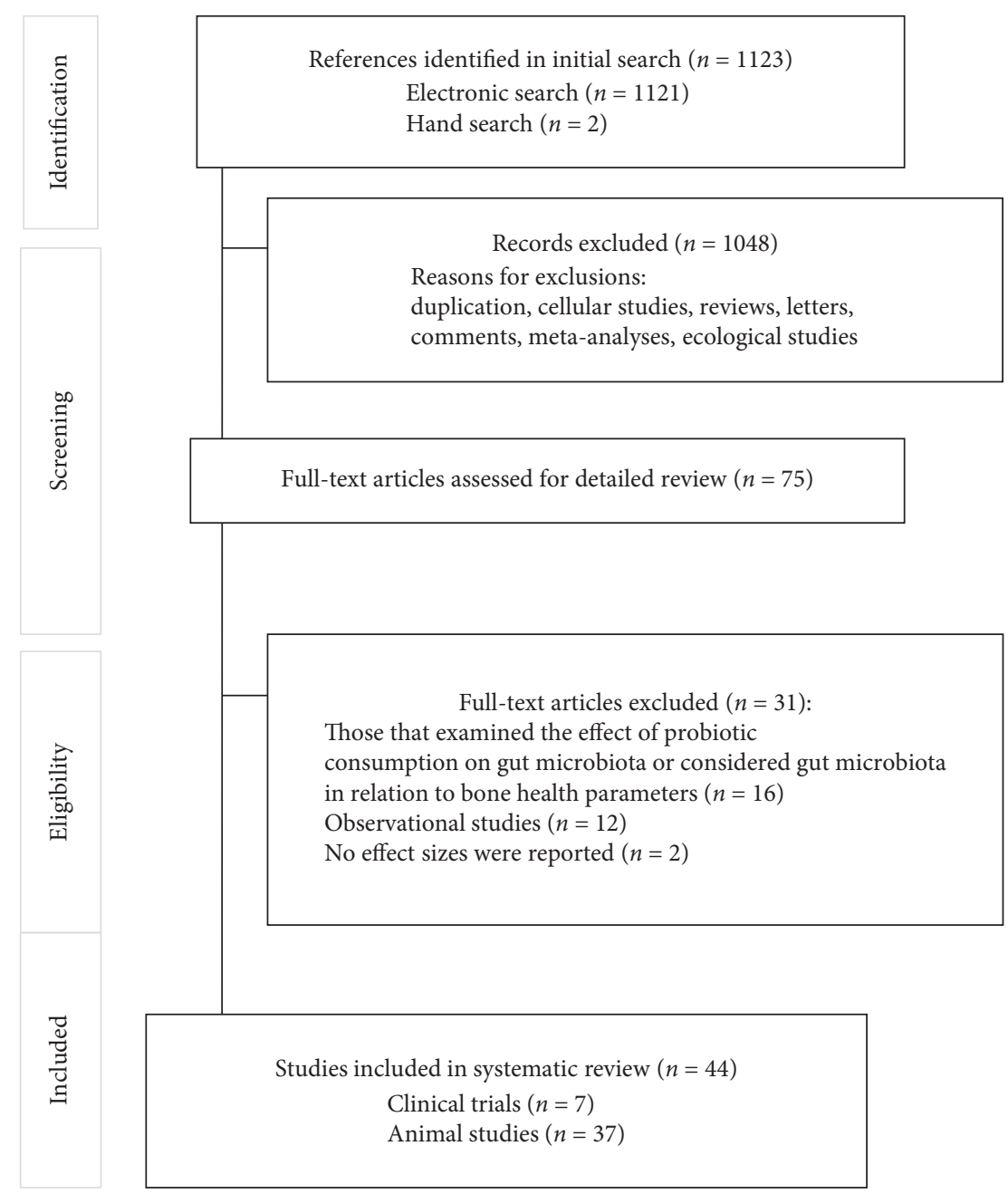

Figure 1: The flow diagram of study selection.

$10^{10} \mathrm{CFU}$ of probiotics per dose. Several types of probiotics were consumed: L. helveticus [21], L. reuteri [68, 69], B. subtilis [19], and combination of various species $[20,22,23]$. The duration of intervention was between 1 day [21] and 12 months [20, 68]. Increased calcium [21] and 25OH-D [69] level, and decreased parathyroid hormone $[21,23,70]$, collagen type 1 cross-linked C-telopeptide (CTX) $[20,23]$, and bone-specific alkaline phosphatase (BALP) [23] level were demonstrated in these publications. Also, increased total hip BMD [19], and reduced BMD loss in L2-L4 [20], femoral neck [20], trochanter [20], and tibia [68] were indicated in these investigations. Almost all of the publications had high-quality score according to the Cochrane quality assessment tool for RCTs, except for one of them [22]. This study assessed the effect of symbiotic and probiotic consumption and BMC. Due to lack of effect sizes, we could not perform meta-analyses in BMC. Therefore, all high-quality score studies were included in different meta-analyses.

3.3. Meta-Analysis. Some chemical parameters such as level of serum calcium, serum phosphorus, $\mathrm{PTH}$, and urinary calcium had enough effect sizes (at least 3 effect sizes) to perform a meta-analysis to calculate combined results of probiotic consumption on bone health parameters. Most of clinical trials in this regard assessed the relation between probiotic consumption and bone health parameters in women 50 years and older. Combining four effect sizes of three studies indicated that probiotic consumption had significantly increased serum calcium levels (weighted mean difference (WMD): $3.82 \mathrm{mmol} / \mathrm{l} ; 95 \%$ CI: 1.05, $6.59 \mathrm{mmol} / \mathrm{l}$; $I$-square $=98.0 \%, P<0.0001) \quad($ Figure 2(a) $)[21,23,69]$. Although significant heterogeneity was reported, low number of included studies did not let us to perform subgroup analysis and find source of heterogeneity. Combining four effect sizes of three studies, we did not find any significant effect of probiotic consumption on serum phosphorus levels (WMD: $1.14 \mathrm{mmol} / \mathrm{l} ; \quad 95 \%$ CI: -0.44 , $2.73 \mathrm{mmol} / \mathrm{l}$ ) (Figure 2(b)) [21, 23, 69]. In terms of PTH levels, probiotic consumption significantly decreases PTH levels (WMD: $-5.53 \mathrm{ng} / \mathrm{l} ;$ 95\%CI: $-9.83,-0.86 \mathrm{ng} / \mathrm{l}, \quad I-$ square $=98.2 \%, P<0.0001) \quad($ Figure $3(\mathrm{a}))[21,23]$. Combining three effect sizes of two studies, we found that probiotic consumption significantly influences urinary calcium levels (WMD: $4.85 \mathrm{mmol} / \mathrm{l} ; 95 \%$ CI: 1.16 , 


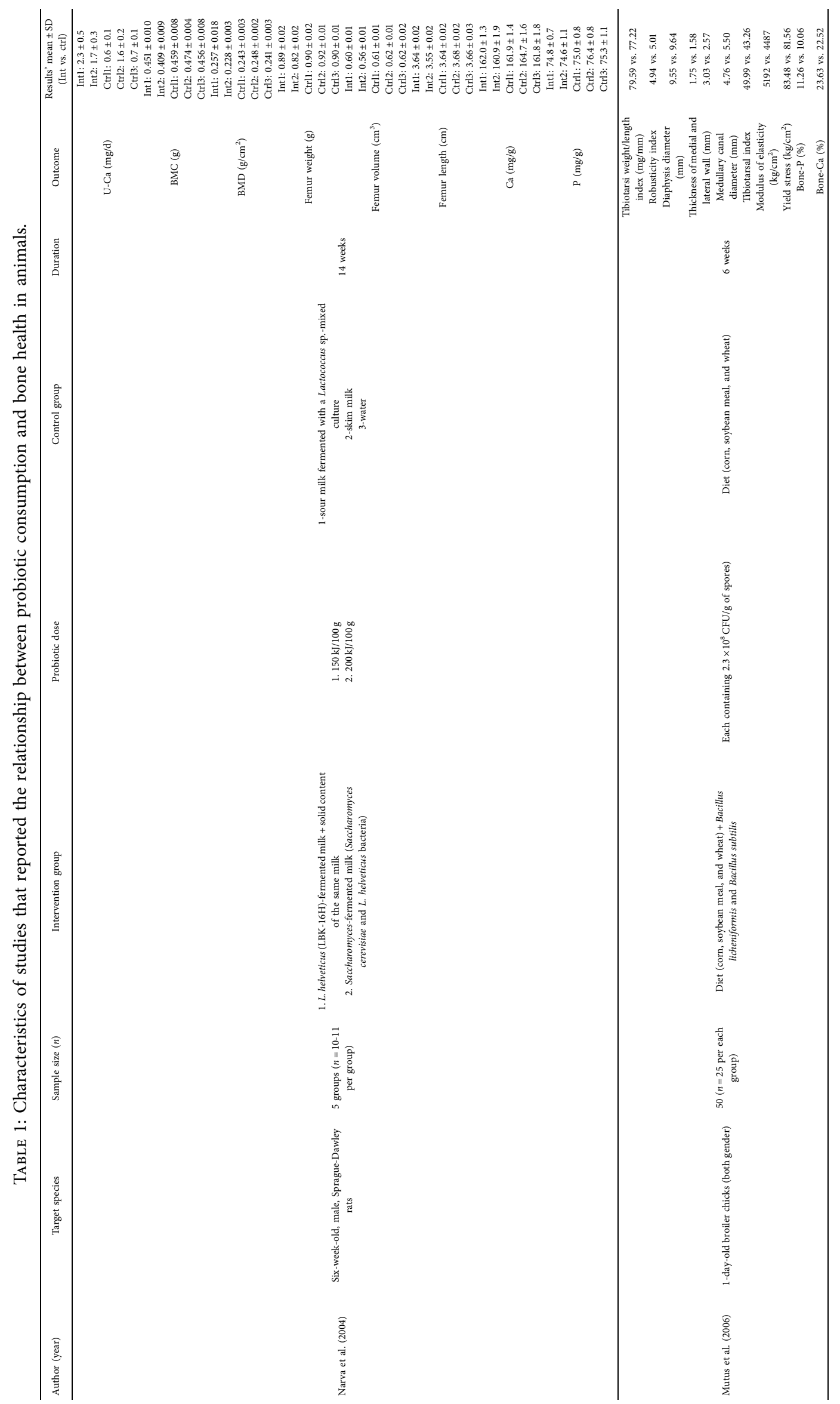




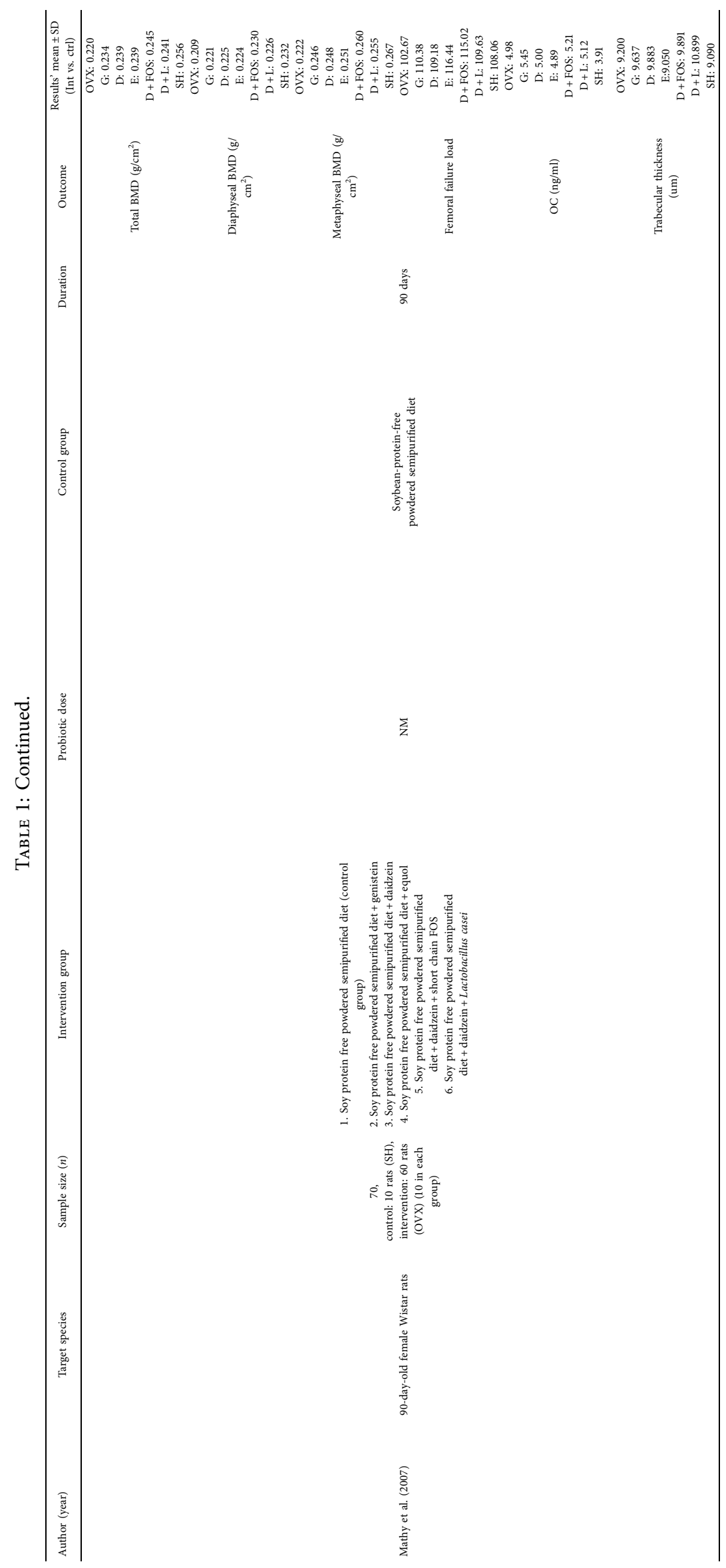




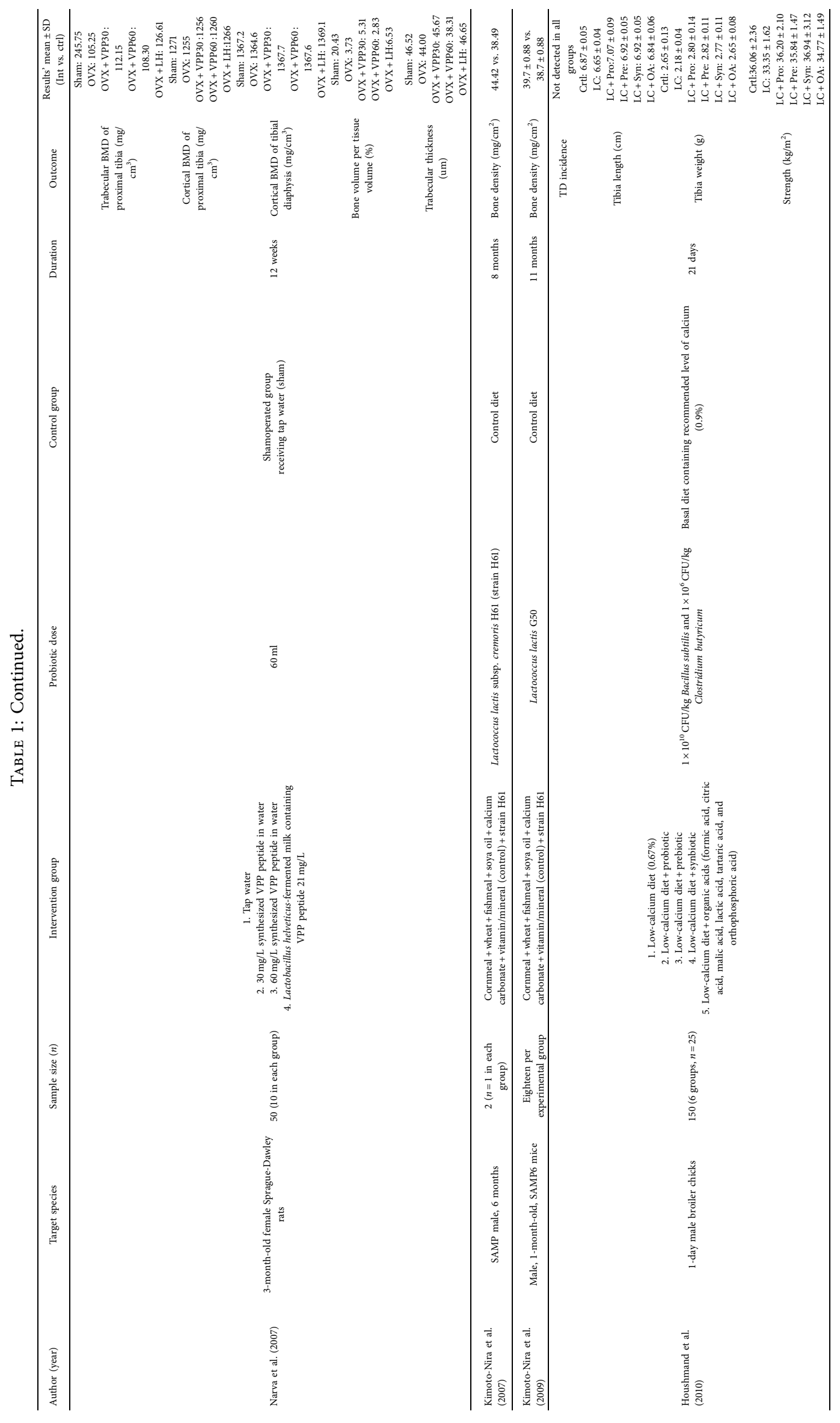




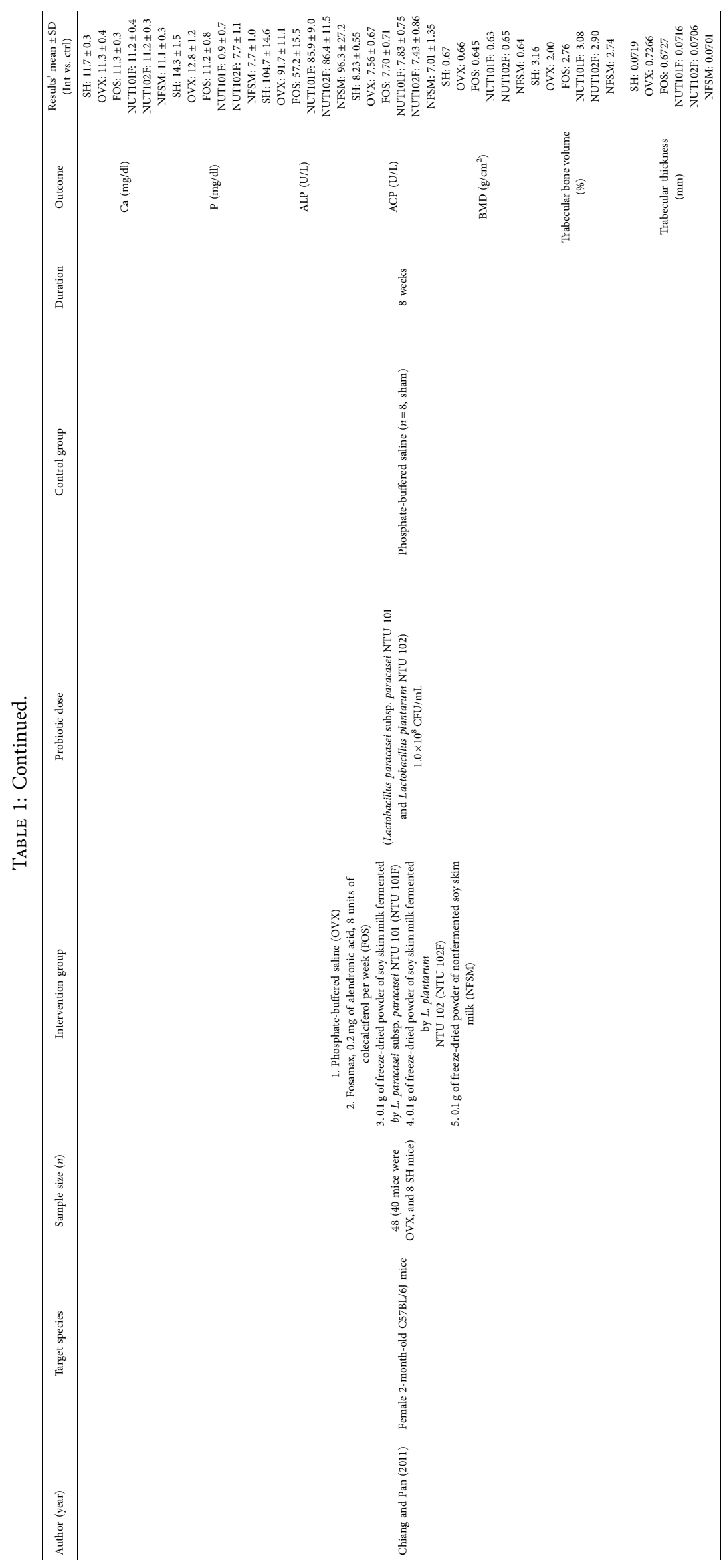




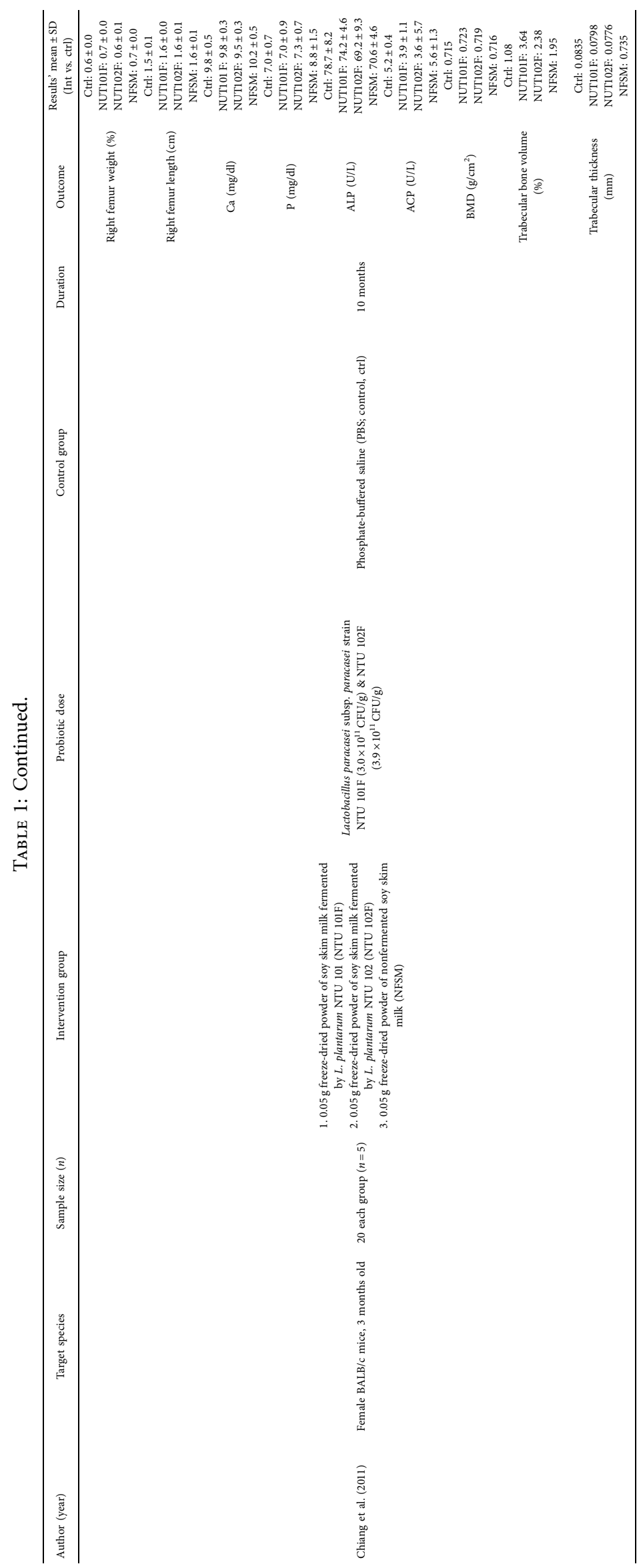




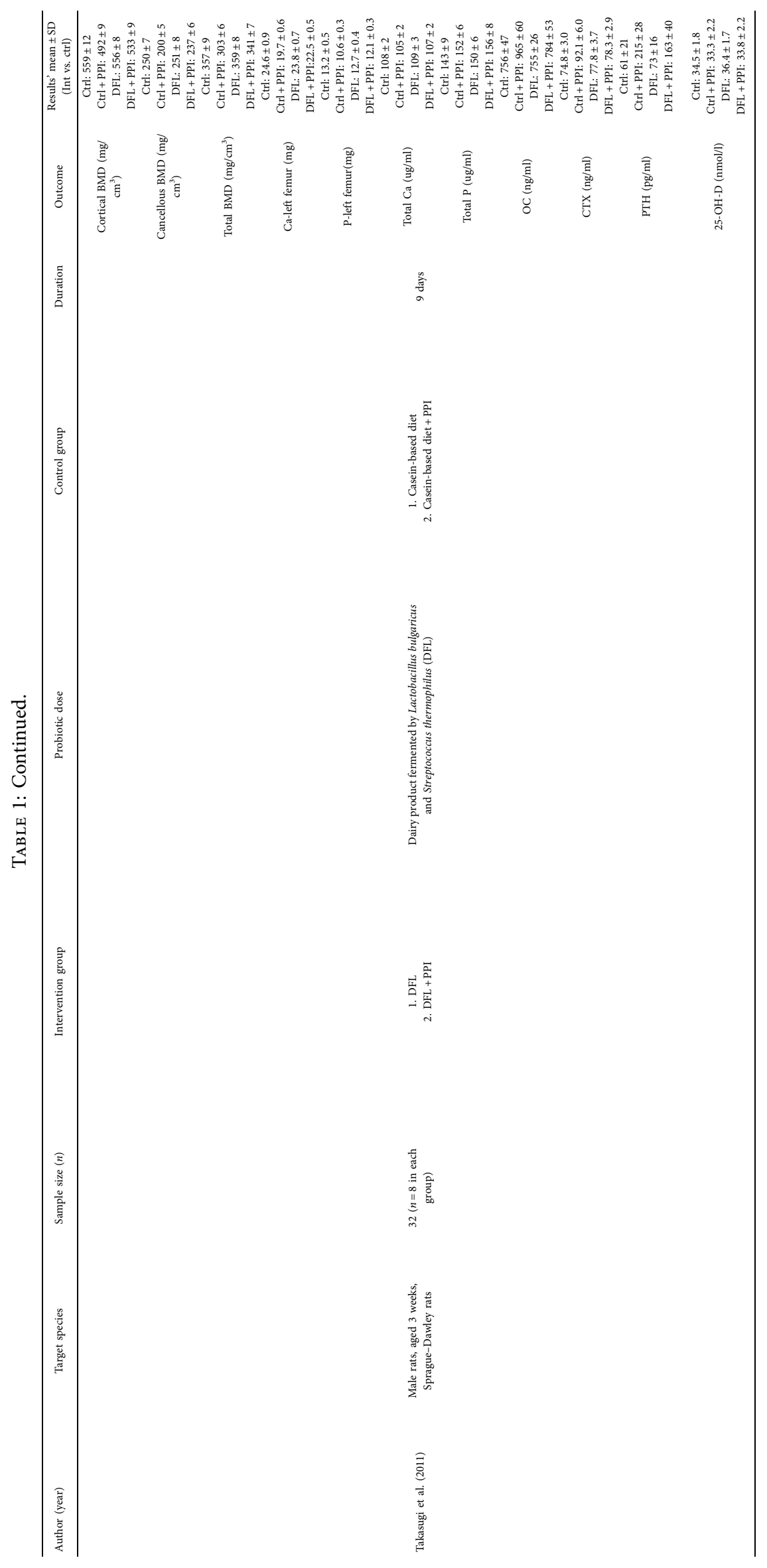




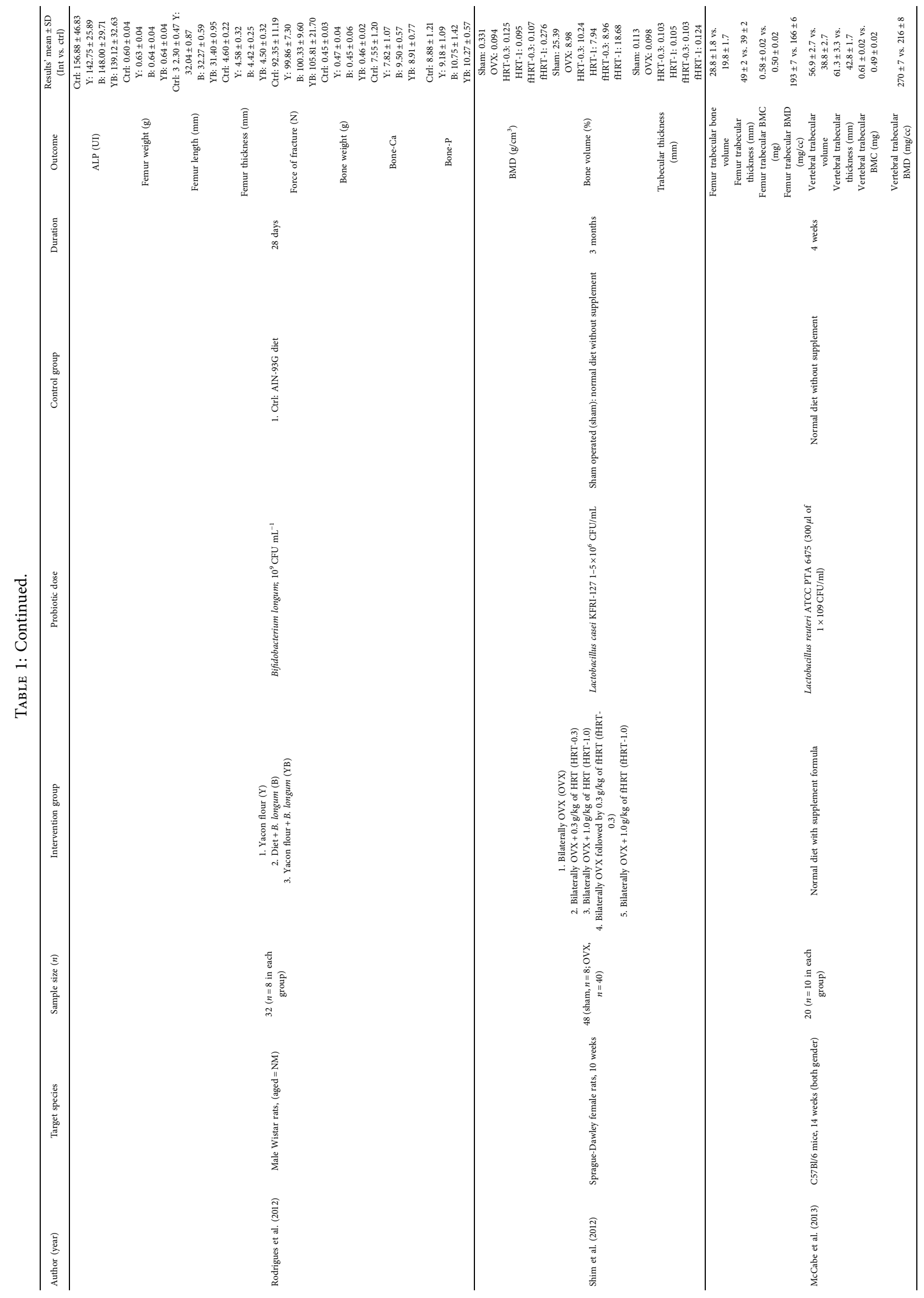




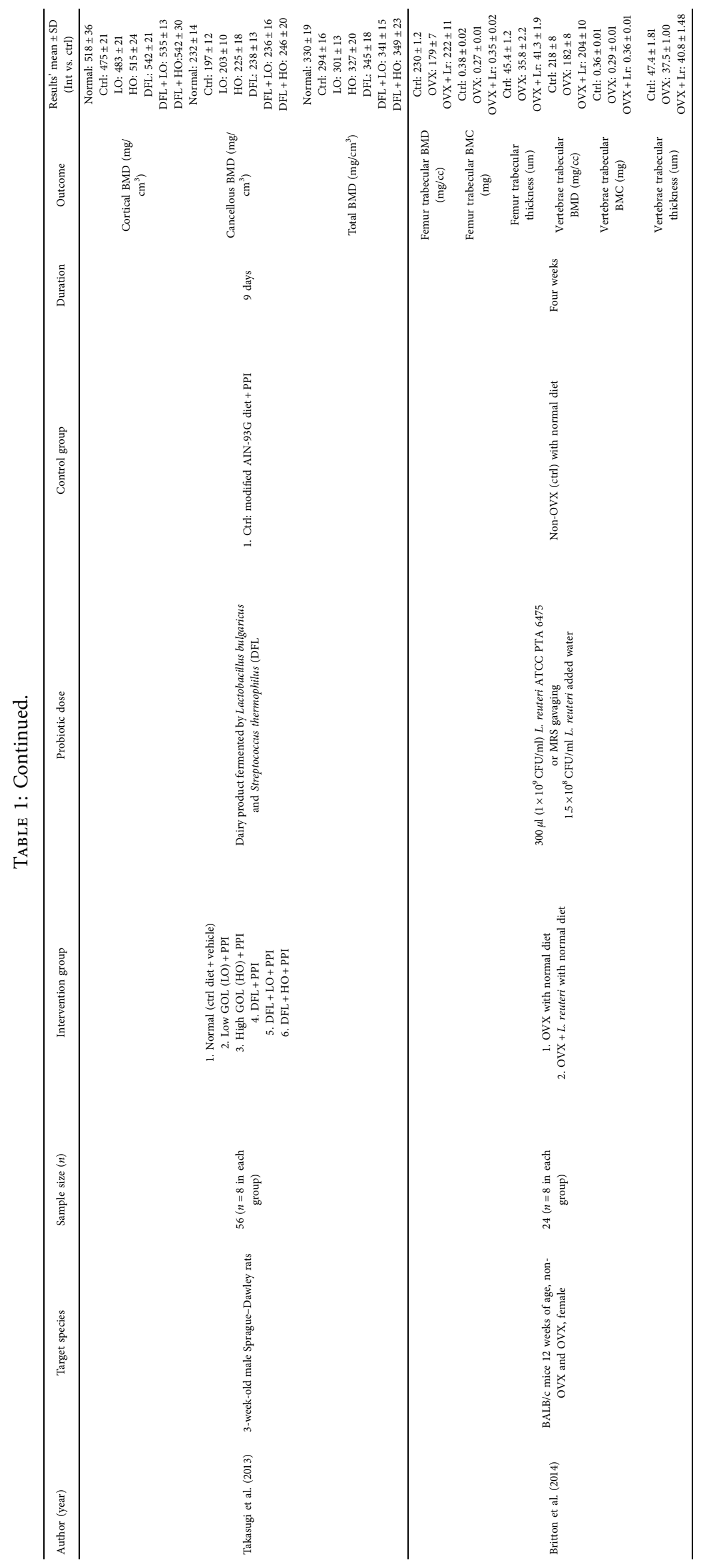




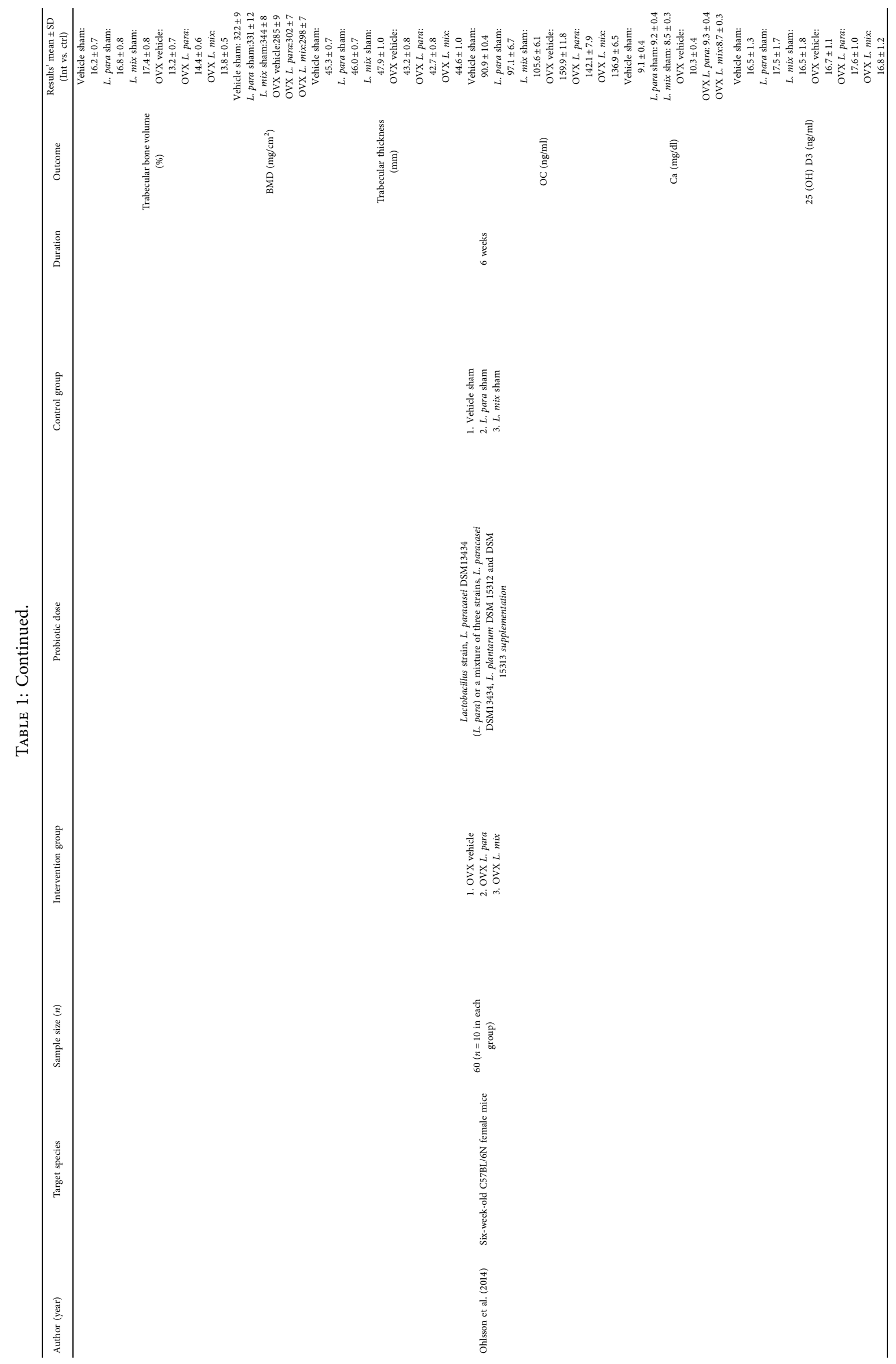




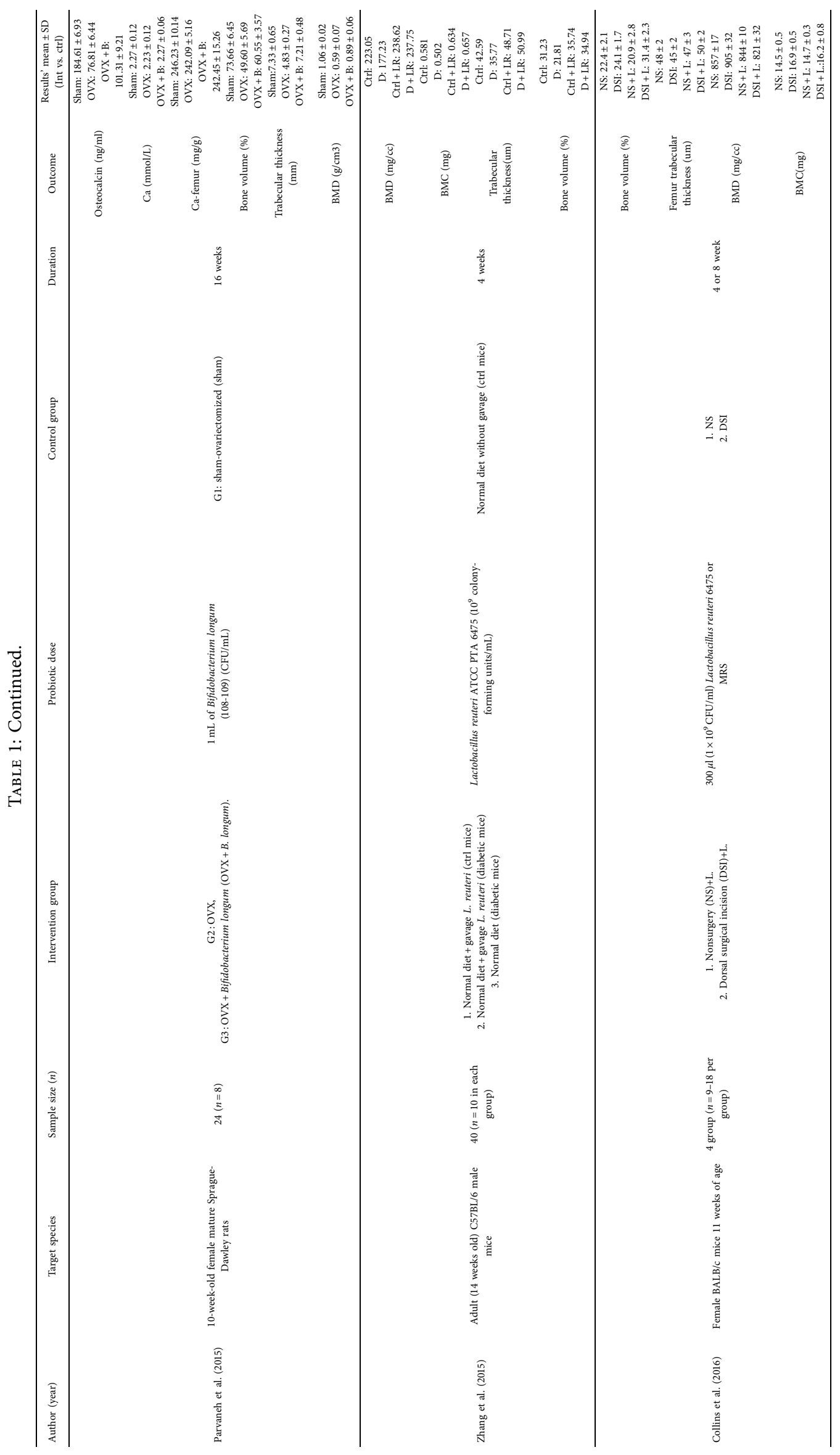




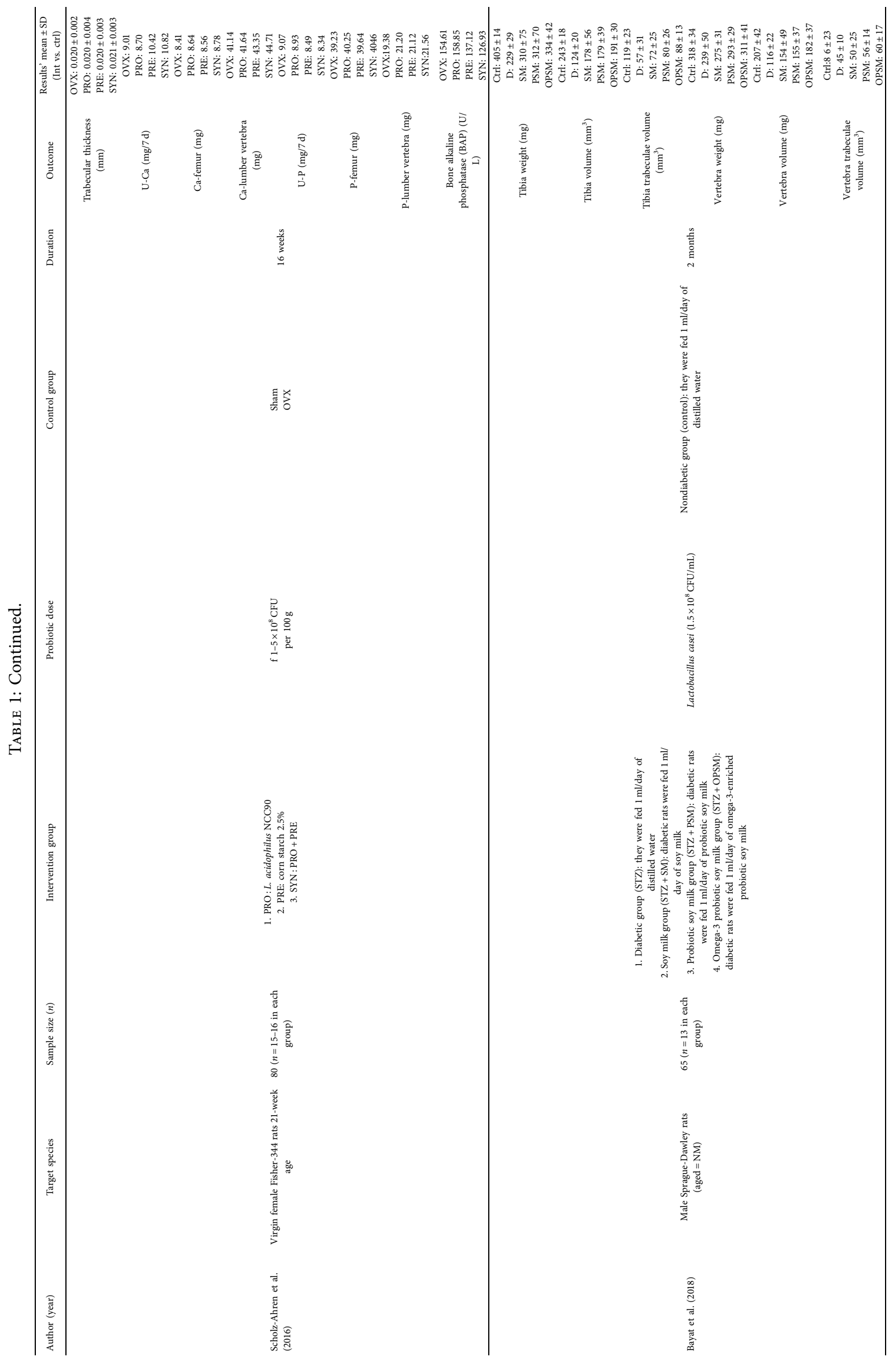




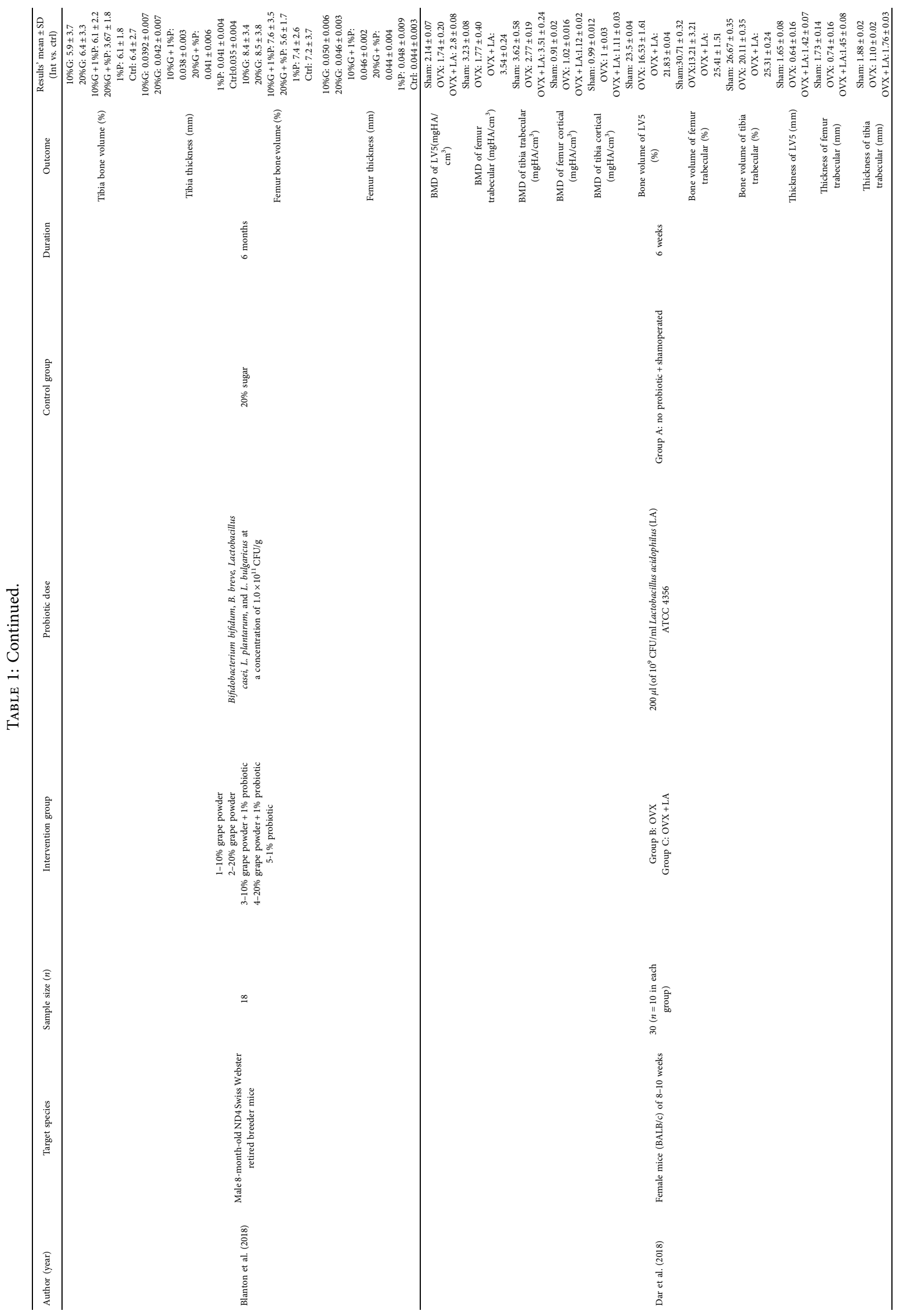




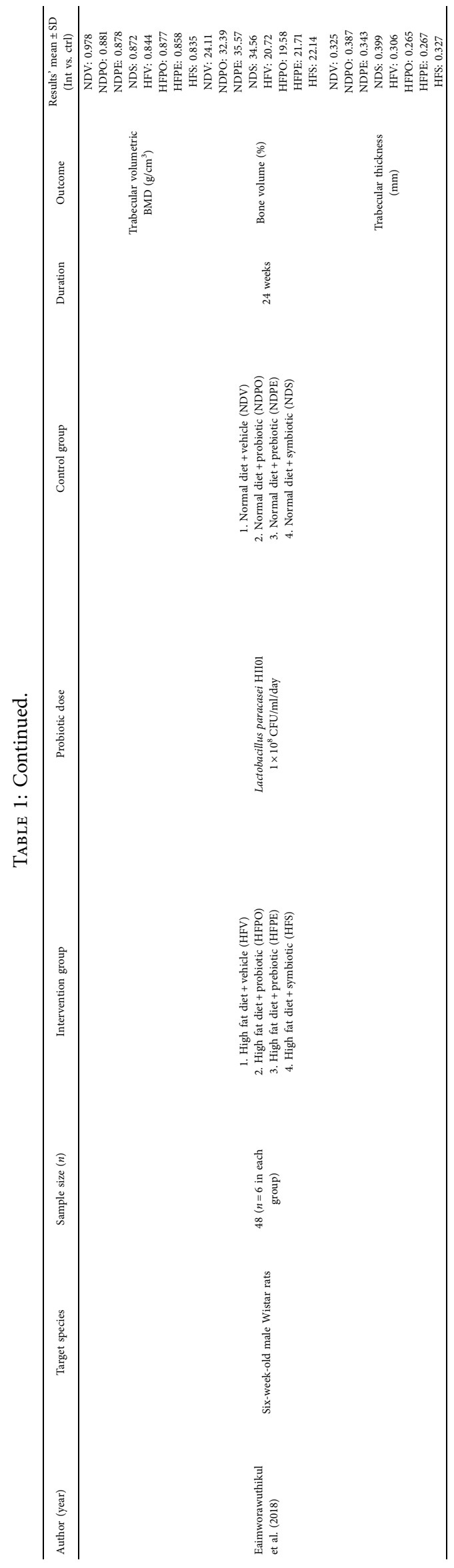




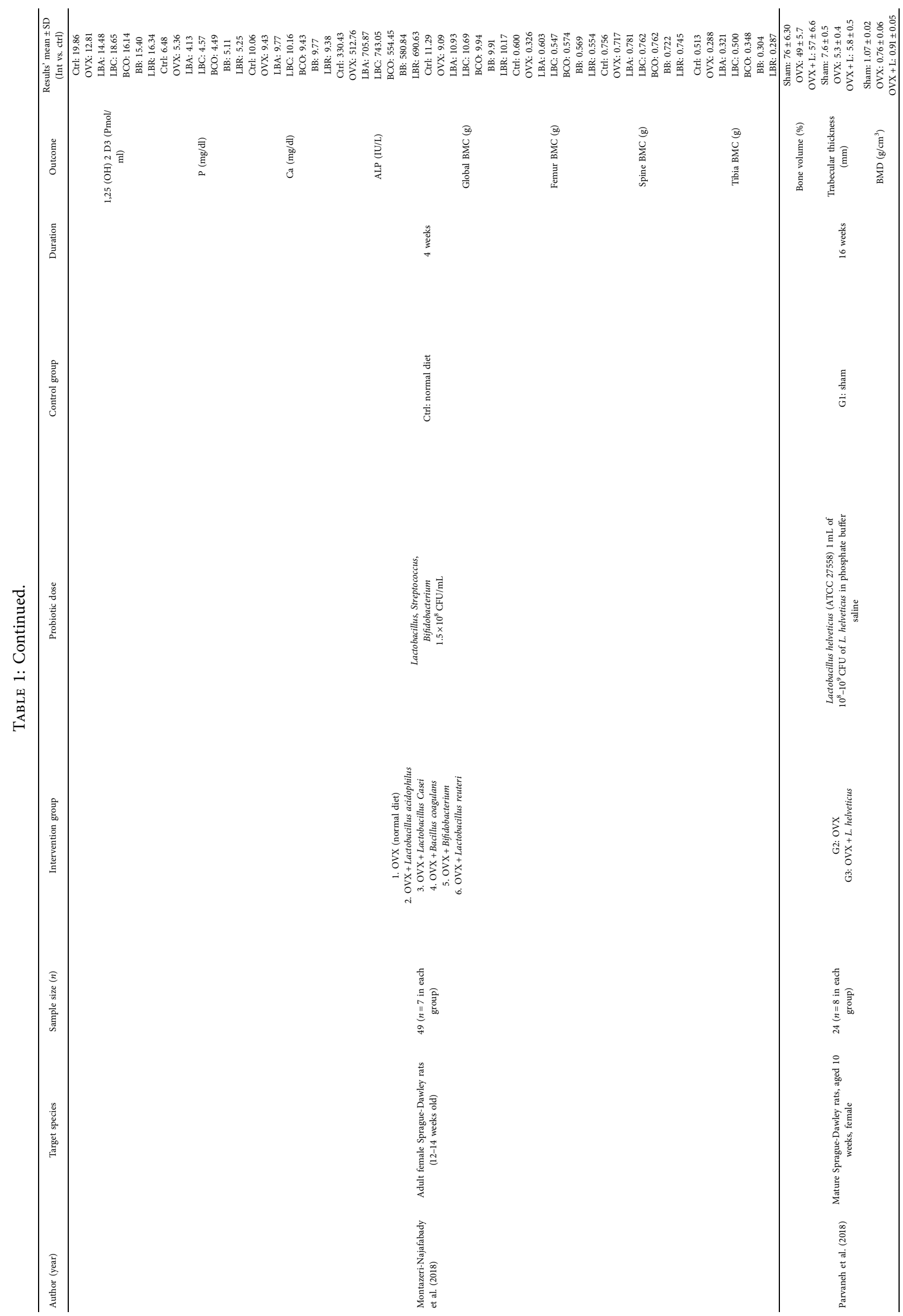




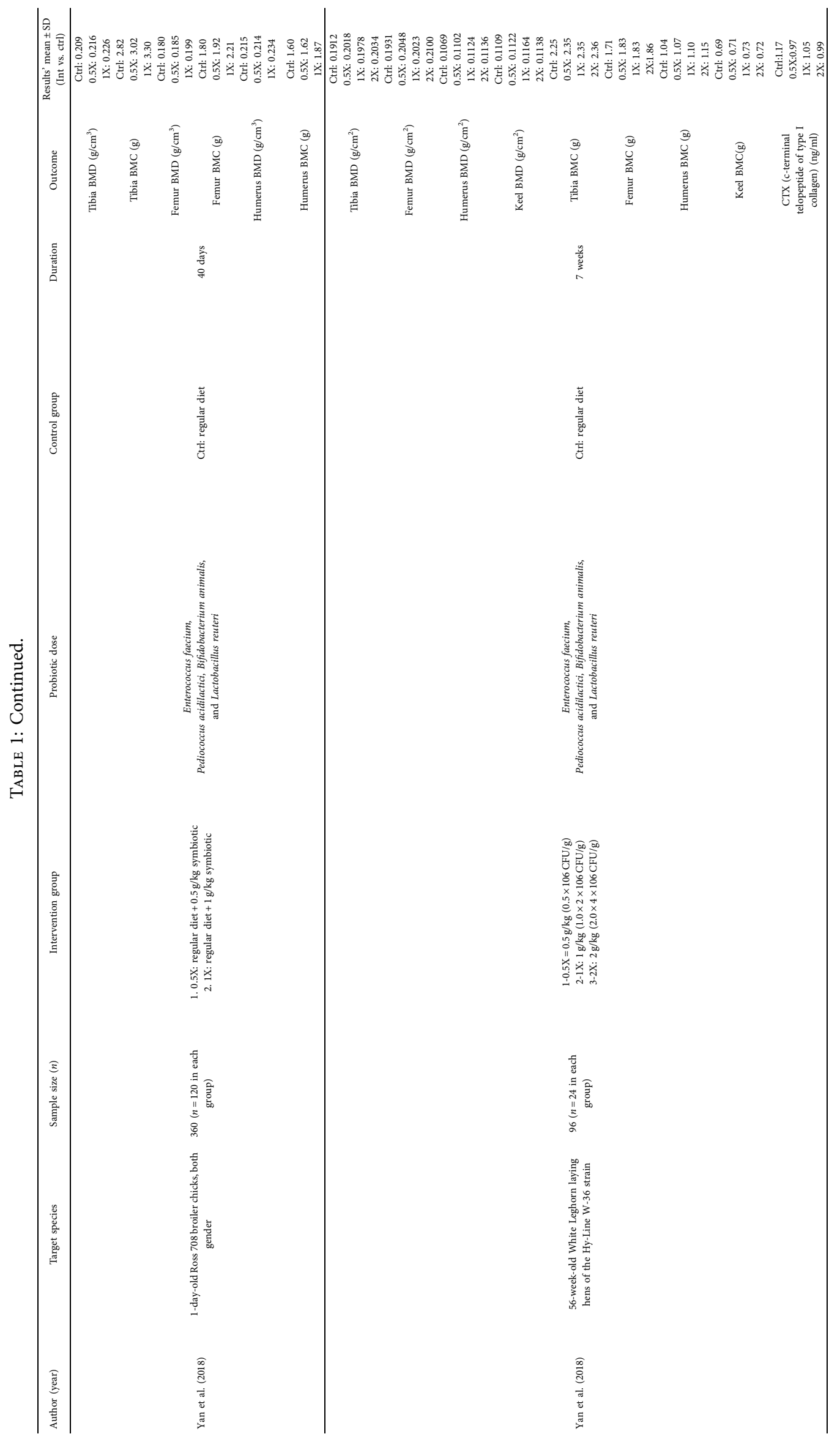




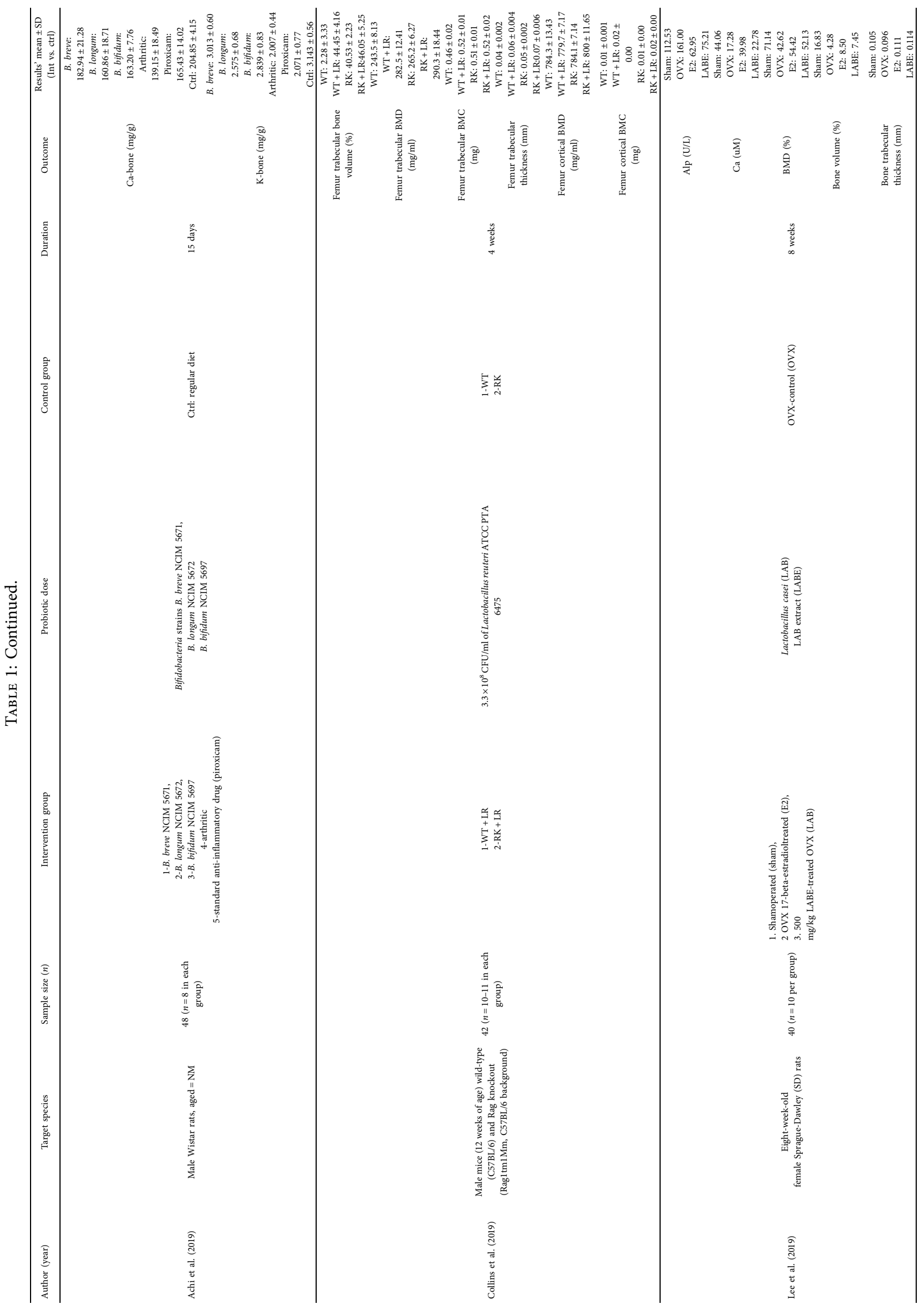




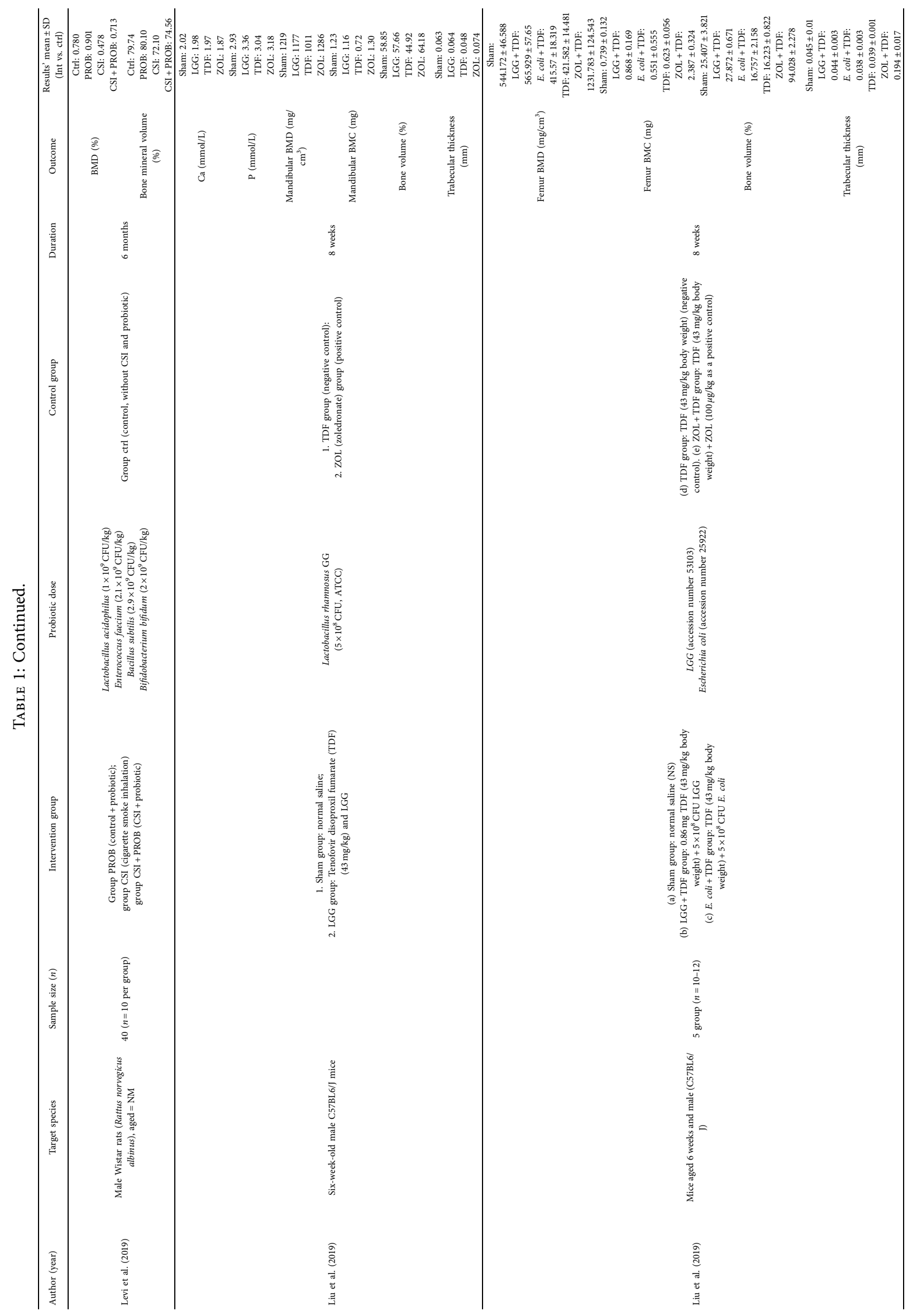




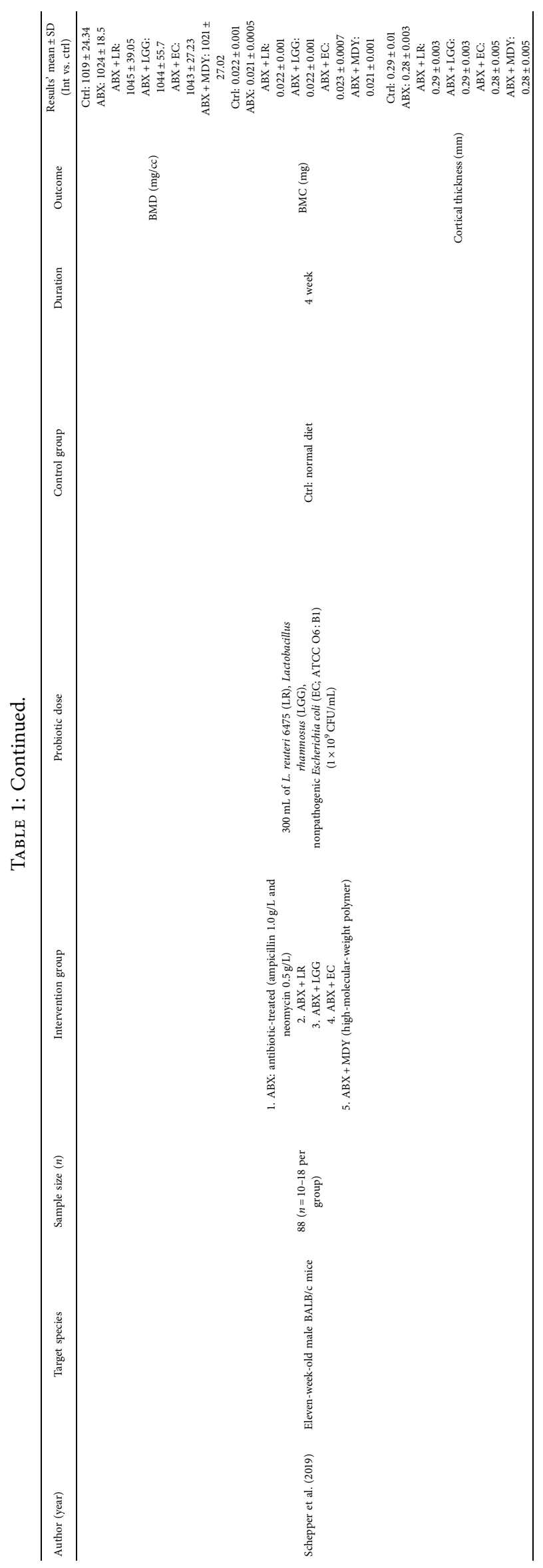




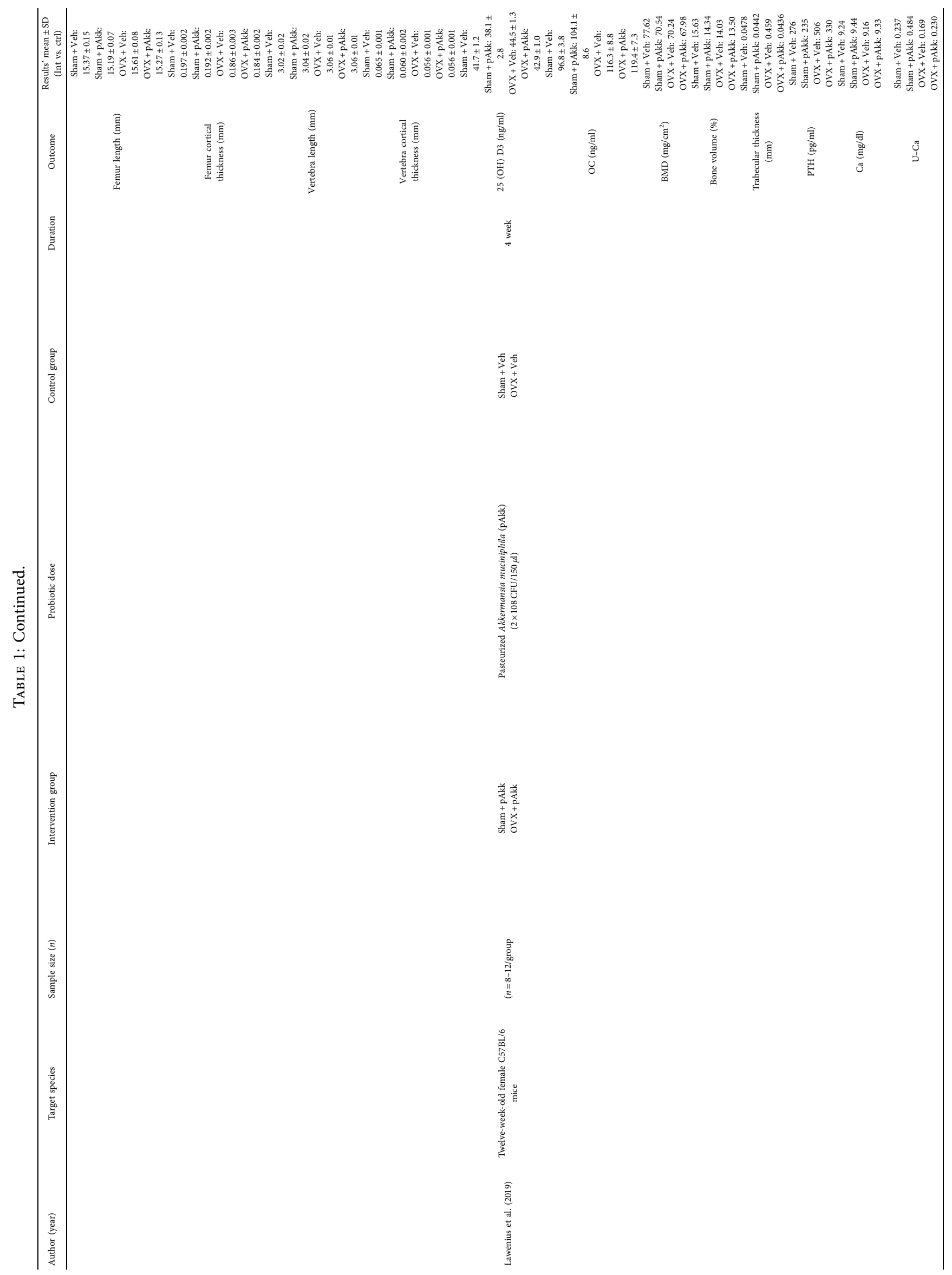




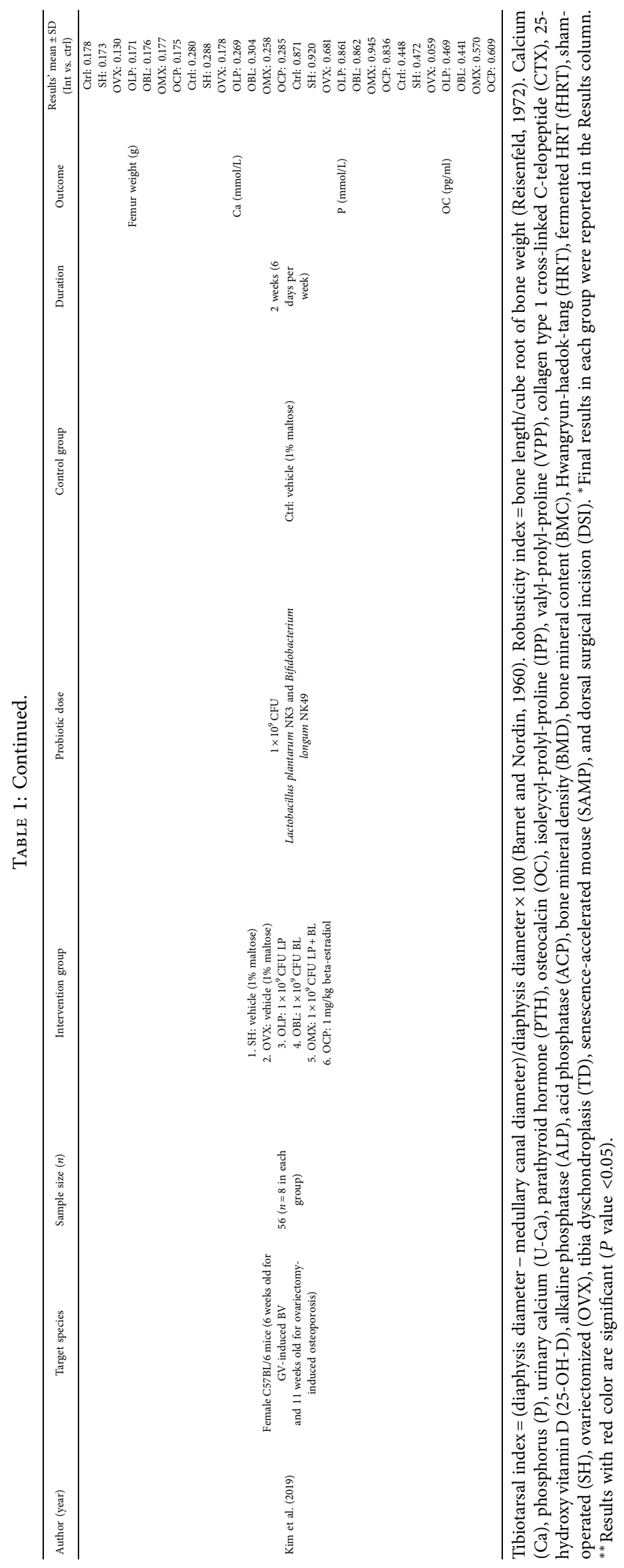




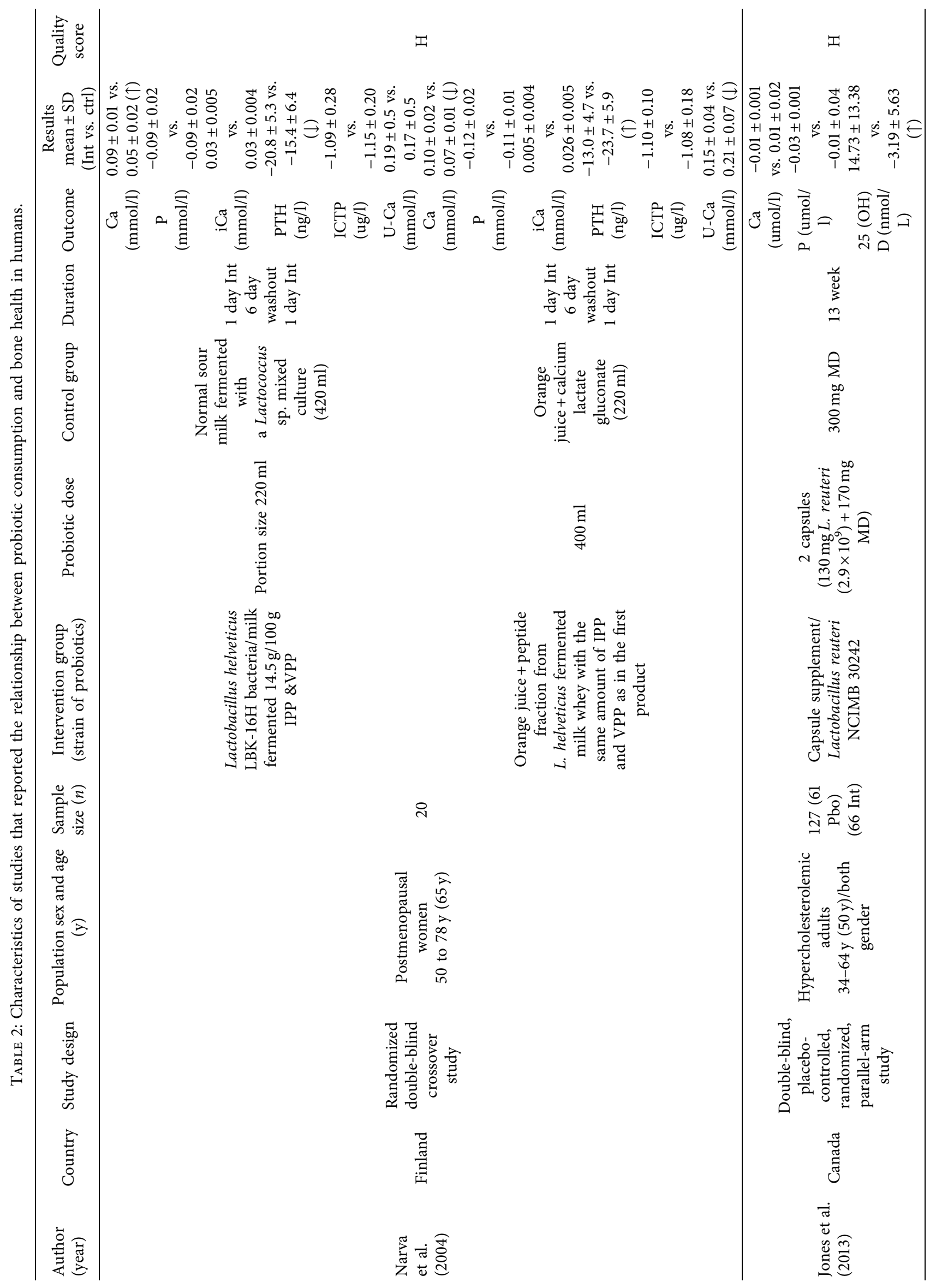




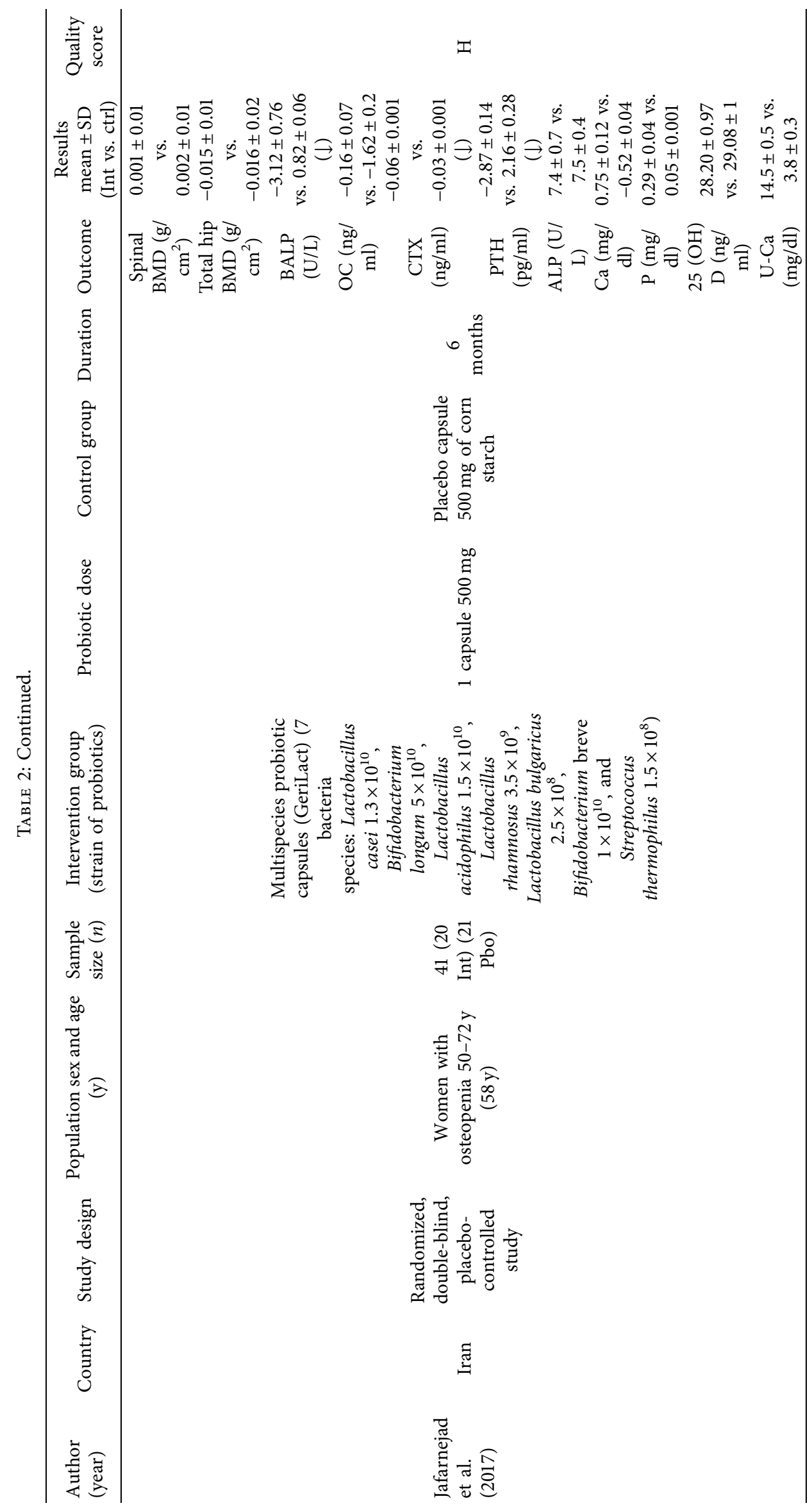




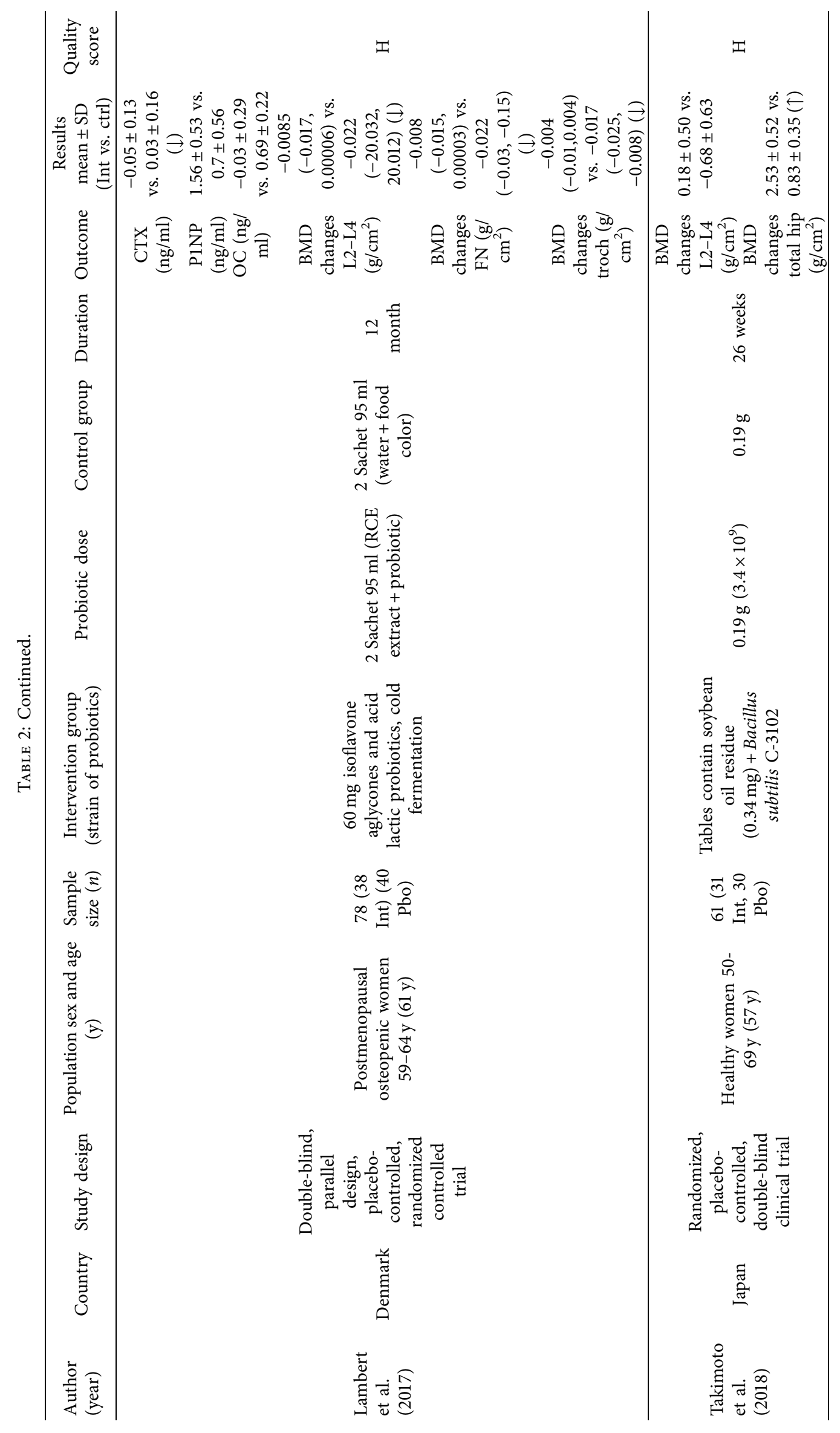




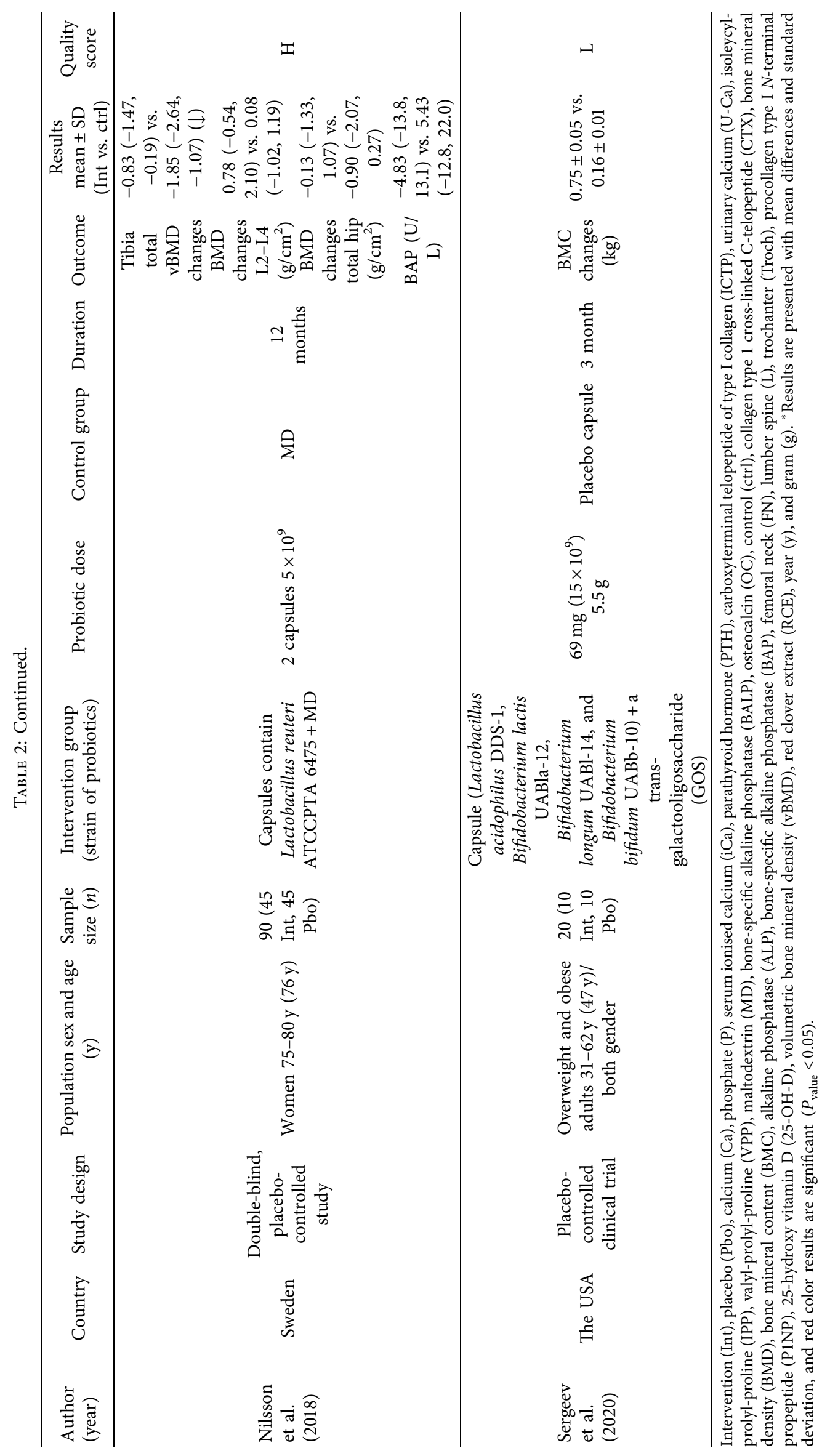




\begin{tabular}{|c|c|c|c|c|}
\hline \multicolumn{3}{|l|}{ Author (year) } & \multirow{2}{*}{$\begin{array}{l}\operatorname{SMD}(95 \% \mathrm{CI}) \\
2.53(1.69,3.37)\end{array}$} & \multirow{2}{*}{$\begin{array}{c}\text { Weight (\%) } \\
26.51\end{array}$} \\
\hline Narva (2004) & $\rightarrow$ & & & \\
\hline Narva (2004) & - & & $1.90(1.14,2.65)$ & 26.64 \\
\hline Jones (2013) & - & & $-0.72(-1.08,-0.36)$ & 27.05 \\
\hline Jafarnejad (2017) & & & $14.35(11.10,17.59)$ & 19.80 \\
\hline Overall $(I$-squared $=98.0 \%, p=0.000)$ & & & $3.82(1.05,6.59)$ & 100.00 \\
\hline Note: weights are from random effects analysis & & & & \\
\hline-17.6 & 0 & 17.6 & & \\
\hline
\end{tabular}

(a)

\begin{tabular}{|c|c|c|}
\hline Author (year) & \multirow{2}{*}{$\frac{\operatorname{SMD}(95 \% \mathrm{CI})}{0.00(-0.62,0.62)}$} & \multirow{2}{*}{$\begin{array}{c}\text { Weight (\%) } \\
25.90\end{array}$} \\
\hline Narva (2004) & & \\
\hline Narva (2004) & $6.20(4.67,7.72)$ & 21.56 \\
\hline Jones (2013) & $-0.72(-1.08,-0.36)$ & 26.61 \\
\hline Jafarnejad (2017) & $0.00(-0.61,0.61)$ & 25.93 \\
\hline Overall $(I$-squared $=96.1 \%, p=0.000)$ & $1.14(-0.44,2.73)$ & 100.00 \\
\hline Note: weights are from random effects analysis & & \\
\hline-7.72 & 7.72 & \\
\hline
\end{tabular}

(b)

Figure 2: Forest plots for the effect of probiotic consumption on (a) serum calcium levels and (b) serum phosphorus levels, expressed as mean differences between intervention and the control diets.

$8.53 \mathrm{mmol} / \mathrm{l} ; \quad I$-square $=97.6 \%, P<0.0001) \quad($ Figure $3(\mathrm{~b}))$ $[21,23]$.

Bone mineral density was calculated in different locations such as spinal, total hip, femoral neck, and troch. BMD at spinal and total hip had enough effect sizes, and we performed meta-analysis in these parameters. Combining four effect sizes of three studies indicated that probiotic consumption did not influence spinal BMD levels (WMD: $0.65 \mathrm{~g} / \mathrm{cm}^{2} ; 95 \%$ CI: $-0.18,1.47 \mathrm{~g} / \mathrm{cm}^{2}$ ) (Figure $4(\mathrm{a})$ ) $[19,20,23,68]$. In terms of total hip BMD, combining three effect sizes of three studies had shown nonsignificant increase in BMD level of total hip (WMD: $1.45 \mathrm{~g} / \mathrm{cm}^{2}$; $95 \% \mathrm{CI}$ : $-0.38,3.28 \mathrm{~g} / \mathrm{cm}^{2}$ ) (Figure 4(b)) $[19,23,68]$.

3.4. Sensitivity Analysis. To investigate the influence of each individual study on the overall findings, we excluded studies from the analysis, stage by stage, and found no significant impact of any individual study on the overall effect sizes.

3.5. Publication Bias. The funnel plots indicated moderate asymmetry, suggesting that publication bias cannot be completely excluded as a factor of influence on the present meta-analysis (data not shown). However, Begg's and
Egger's regression tests provided no evidence of substantial publication bias.

\section{Discussion}

In this systematic review, we found some effects of probiotic supplementation on bone health parameters such as serum and urinary calcium levels and PTH levels. Some strains of Bifidobacterium and Lactobacillus such as L. reuteri, L. casei, L. paracasei, L. bulgaricus, and L. acidophilus indicated beneficial effects on bone health parameters in animal experiments and clinical trials.

Probiotic consumption has been assessed in few clinical trials. In most of them, probiotic consumption had beneficial effects on bone health parameters such as BMD, serum calcium, $25(\mathrm{OH}) \mathrm{D}$, and PTH levels. Only one clinical trial in this regard had reported no effect of probiotic consumption on bone health parameters that has been done on overweight and obese adults [22]. There are some critical points, which should be considered in the interpretation of the results of this study. This study had no randomization or blinding, so we cannot rule out the probable risk of bias. Notably, participants were obese or overweight, and we all know that this condition could change gut microbiota $[71,72]$ as well as hormonal status [70]. Considering all 


\begin{tabular}{|c|c|c|c|}
\hline Author (year) & & SMD $(95 \% \mathrm{CI})$ & Weight (\%) \\
\hline Narva (2004) & & $-0.92(-1.57,-0.27)$ & 37.31 \\
\hline Narva (2004) & + & $2.01(1.24,2.77)$ & 37.20 \\
\hline Jafarnejad (2017) & & $-22.55(-27.59,-17.51)$ & 25.49 \\
\hline Overall $(I$-squared $=98.2 \%, p=0.000)$ & & $-5.35(-9.83,-0.86)$ & 100.00 \\
\hline \multicolumn{4}{|l|}{ Note: weights are from random effects analysis } \\
\hline-27.6 & 0 & 27.6 & \\
\hline
\end{tabular}

(a)

\begin{tabular}{|c|c|c|c|}
\hline Author (year) & & SMD $(95 \% \mathrm{CI})$ & Weight (\%) \\
\hline Narva (2004) & 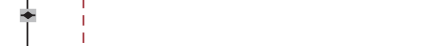 & $0.04(-0.58,0.66)$ & 39.97 \\
\hline Narva (2004) & $\bullet$ & $-1.05(-1.72,-0.39)$ & 39.91 \\
\hline Jafarnejad (2017) & - & $26.10(20.27,31.92)$ & 20.12 \\
\hline Overall $(I$-squared $=97.6 \%, p=0.000)$ & & $4.85(1.16,8.53)$ & 100.00 \\
\hline Note: weights are from random effects analysis & I & & \\
\hline-31.9 & 31.9 & & \\
\hline
\end{tabular}

(b)

FIGURE 3: Forest plots for the effect of probiotic consumption on (a) PTH levels and (b) urinary calcium levels, expressed as mean differences between intervention and the control diets.

clinical trials, it seems that the consumption of probiotics may have positive effects on bone health in humans. Metaanalysis indicated that probiotic consumption improved some bone health parameters such as serum calcium levels and PTH. Significant heterogeneity is reported in our findings. Due to the small number of effect sizes, we could not use subgroup analyses to find the source of heterogeneity. Although studies in this area had high quality, they had some limitation. There are some differences between the previous included publications that could be the cause of heterogeneity. It seems that the mean age of the participants, gender, bone health status, and chronic conditions can justify the heterogeneity between studies.

Compared to clinical trials, the effects of probiotics on bone health have been addressed more in the animal experiments. Although in eight articles no effects of probiotics on bone health parameters were detected, most of the previous publications had reported beneficial effects of probiotic feeding on the bone health status of animals. There are several points that could explain the lack of connection between probiotic feeding and bone health parameters in these eight articles. Species of rats included in these surveys are different; virgin fisher rat [58], SAMP rat [60, 61], and ND4 Swiss Webster retired breeder mice [63] were used, while other studies used Sprague-Dawley rat, C57BL/6J mice, Wistar rat, and BALB/c mice. Sample sizes in one of these studies are very low (one rat in each group [60]), and older rats were included in these studies [37, 46, 47]. Considering the limitations of studies, it seems that probiotic feedings have beneficial effects on bone health parameters in animal experiments.

Gut microbiota is considered as an organ involving in mucosal barrier function, immune system, endocrine system, food digestion, and energy metabolism as well as bone health and metabolism [73-77]. Gut microbiota could regulate bone metabolism through the effects on the immune system, the endocrine organs, and calcium absorption. Some species of intestinal bacteria promote the release of inflammatory mediators, such as tumor necrosis factor- $\alpha$ (TNF $\alpha$ ), interleukin (IL)-1, and IL-6 which plays an important role in the formation of osteoclasts and osteoblasts [78]. Intestinal microbiota also promotes the release of endothelial nitric oxide synthase (eNOS). eNOS mRNA regulates the production of osteoblasts and osteoclasts, as well as inflammatory mediators. NO has dichotomous biological effects, and at low concentrations, NO may promote proliferation, differentiation, and survival of osteoblasts, whereas at high concentrations, NO may inhibit bone 


\begin{tabular}{|c|c|c|}
\hline Author (year) & SMD $(95 \% \mathrm{CI})$ & Weight (\%) \\
\hline Jafarnejad (2017) & $-0.10(-0.71,0.51)$ & 24.15 \\
\hline Lambert (2017) & $0.00(-0.44,0.44)$ & 25.78 \\
\hline Takimot (2018) & $1.94(1.33,2.55)$ & 24.16 \\
\hline Nilsson (2018) & $0.78(0.35,1.20)$ & 25.91 \\
\hline Overall $(I$-squared $=90.3 \%, p=0.000)$ & $0.65(-0.18,1.47)$ & 100.00 \\
\hline Note: weights are from random effects analysis & & \\
\hline-2.55 & 2.55 & \\
\hline
\end{tabular}

(a)

\begin{tabular}{|c|c|c|c|}
\hline Author (year) & & SMD $(95 \%$ CI $)$ & Weight (\%) \\
\hline Jafarnejad (2017) & & $0.06(-0.55,0.68)$ & 33.49 \\
\hline Takimot (2018) & & $3.82(2.97,4.68)$ & 32.35 \\
\hline Nilsson (2018) & $\longrightarrow$ & $0.57(0.15,0.99)$ & 34.16 \\
\hline Overall $(I$-squared $=96.3 \%, p=0.000)$ & & $1.45(-0.38,3.28)$ & 100.00 \\
\hline Note: weights are from random effects analysis & & & \\
\hline-4.68 & 0 & .68 & \\
\hline
\end{tabular}

(b)

FIGURE 4: Forest plots for the effect of probiotic consumption on (a) spinal BMD levels and (b) total hip BMD levels, expressed as mean differences between intervention and the control diets.

resorption and formation. Therefore, at a certain concentration range, $\mathrm{NO}$ can avoid osteoclast-mediated bone resorption and promote osteoblast growth $[79,80]$. Probiotics could affect gut microbiota and regulate immune cells and inflammatory cytokines or hormones and growth factors by inducing the host's production of $\beta$-defensin and $\operatorname{IgA}$. Probiotics may also be able to enhance the intestinal barrier function by maintaining tight junctions and inducing mucin production. Probiotic-mediated immunomodulation may occur through the mediation of cytokine secretion signaling pathways such as NFKB and MAPKs, which plays a vital role in the formation of osteoclasts and osteoblasts [81-83]. Gut microbiota has also critical effects on the endocrine system. Levels of serum IGF-1 can promote the differentiation and growth of bone cells, including osteoblasts and chondrocytes, and enhance normal interactions among them [84]. Moreover, the IGF-1 signaling pathway is involved in the regulation of bone metabolism via both growth hormone $(\mathrm{GH})$ and PTH which directly and indirectly have effects on bone growth [85]. PTH is secreted from parathyroid glands which regulate calcium levels by increasing absorption of calcium in gut, decreasing calcium absorption in kidney and increasing bone resorption. Bone remodeling is a dynamic coordination process between bone formation with osteoblasts and resorption with osteoclasts [86]. Increasing PTH level leads to more bone destruction by osteoclasts. As we indicated in meta-analysis results, greater calcium levels reduced PTH levels and osteoclast activity. Moreover, gonadal steroids, including estrogen and androgen, play key roles in the regulation of bone mass and turnover [87]. Gut microbiota regulates bone metabolism by affecting the absorption of calcium. Calcium absorption can be facilitated by vitamin $\mathrm{D}$. It has been shown that a low-calcium diet alone can lead to bone resorption, high bone turnover, and impaired bone trabecular microarchitecture in multiple bones. Balanced gut microbiota leads to reduced osteoclast activity and increased osteoblast activity within the bone matrix by these strategies, which ultimately results in increased bone structure, density, and strength [88].

The present study has some strengths and limitations. It is the first study that systematically reviews the relationship between probiotic consumption and bone health parameters. In addition, a comprehensive search strategy was performed and no limitation was applied during the search. Furthermore, it is the first publication that performed meta-analysis on probiotic supplementations and bone health parameters. We considered experimental and clinical studies, and we tried to clarify the mechanism. However, some points need to be considered. Participants in the included clinical trials had different health status, for example, hyperlipidemia or obesity, which might influence the results. Moreover, different 
species and dosage of probiotics were used and it might impress findings. Although different probiotic species have different effects, due to the limited publications we combined findings of all studies. In addition, we could not perform meta-analyses in all parameters because of limited number of effect sizes. High heterogeneity was reported, and subgroup analyses could not be performed. Our findings might be considered as primary findings, and further studies should be designed on different bacterial species and strains.

\section{Conclusion}

In conclusion, in this systematic review, we found that probiotic supplementation containing $L$. reuteri, $L$. casei, L. paracasei, L. bulgaricus, L. acidophilus, and B. subtilis might improve bone health parameters in animal and human studies. Meta-analysis of human studies indicated that probiotic consumption has significantly increased serum and urinary calcium levels and decreased PTH level. Further studies are needed to decide on the appropriate probiotic species, strain, and dosages to improve bone health status.

\section{Data Availability}

The (effect sizes) data used to support the findings of this study are included within the article. The (search strategy) data used to support the findings of this study are also included within the supplementary information file.

\section{Ethical Approval}

The study was ethically approved by the Medical Ethics Committee of the Tehran University of Medical Sciences, Tehran, Iran.

\section{Consent}

Not applicable.

\section{Conflicts of Interest}

The authors declare no conflicts of interest.

\section{Authors' Contributions}

Hanieh Malmir, Hanieh-Sadat Ejtahed, and Shirin HasaniRanjbar contributed in conception, design, statistical analyses, data interpretation, and manuscript drafting. AhmadReza Soroush, Amir-Mohammad Mortazavian, Noushin Fahimfar, Afshin Ostovar, Ahmad Esmaillzadeh, and Bagher Larijani had scientific cooperation. All the authors approved the final manuscript for submission.

\section{Acknowledgments}

The authors are thankful of the team working on this study and all participants who made this experience. Endocrinology and Metabolism Research Institute, Tehran
University of Medical Sciences, Tehran, Iran, financially supported this study.

\section{Supplementary Materials}

Supplementary table 1: search strategies and the number of publications in each electronic database. (Supplementary Materials)

\section{References}

[1] C.-B. Ahn and J.-Y. Je, "Bone health-promoting bioactive peptides," Journal of Food Biochemistry, vol. 43, no. 1, p. e12529, 2019.

[2] T. Sozen, L. Ozisik, and N. Calik Basaran, "An overview and management of osteoporosis," European Journal of Rheumatology, vol. 4, no. 1, pp. 46-56, 2017.

[3] J. Richmond, G. B. Aharonoff, J. D. Zuckerman, and K. J. Koval, "Mortality risk after hip fracture," Journal of Orthopaedic Trauma, vol. 17, no. 1, pp. 53-56, 2003.

[4] O. Johnell and J. A. Kanis, "An estimate of the worldwide prevalence and disability associated with osteoporotic fractures," Osteoporosis International, vol. 17, no. 12, pp. 1726-1733, 2006.

[5] I. S. L. Liem, C. Kammerlander, N. Suhm, S. L. Kates, and M. Blauth, "Literature review of outcome parameters used in studies of geriatric fracture centers," Archives of Orthopaedic and Trauma Surgery, vol. 134, no. 2, pp. 181-187, 2014.

[6] A. J. Bollet, G. Engh, and W. Parson, "Epidemiology of osteoporosis," Archives of Internal Medicine, vol. 116, no. 2, pp. 191-194, 1965.

[7] L. J. Melton, "Epidemiology worldwide," Endocrinology and Metabolism Clinics of North America, vol. 32, no. 1, pp. 1-13, 2003.

[8] L. G. Raisz, "Pathogenesis of osteoporosis: concepts, conflicts, and prospects," Journal of Clinical Investigation, vol. 115, no. 12, pp. 3318-3325, 2005.

[9] F. Ojo, S. Al Snih, L. A. Ray, M. A. Raji, and K. S. Markides, "History of fractures as predictor of subsequent hip and nonhip fractures among older Mexican Americans," Journal of the National Medical Association, vol. 99, no. 4, pp. 412418, 2007.

[10] P. K. K. Wong, J. J. Christie, and J. D. Wark, "The effects of smoking on bone health," Clinical Science, vol. 113, no. 5, pp. 233-241, 2007.

[11] P. Anagnostis, A. Karagiannis, A. I. Kakafika, K. Tziomalos, V. G. Athyros, and D. P. Mikhailidis, "Atherosclerosis and osteoporosis: age-dependent degenerative processes or related entities?" Osteoporosis International, vol. 20, no. 2, pp. 197-207, 2009.

[12] L. R. McCabe and N. Parameswaran, "Advances in probiotic regulation of bone and mineral metabolism," Calcified Tissue International, vol. 102, no. 4, pp. 480-488, 2018.

[13] F. L. Collins, N. D. Rios-Arce, J. D. Schepper, N. Parameswaran, and L. R. McCabe, "The potential of probiotics as a therapy for osteoporosis," Microbiology Spectrum, vol. 5, no. 4, 2017.

[14] E. Thursby and N. Juge, "Introduction to the human gut microbiota," Biochemical Journal, vol. 474, no. 11, pp. 1823-1836, 2017.

[15] A. K. Jain, "Regulation of foods containing probiotics-codex alimentarius, Japan, European union and India," International Journal of Fermented Foods, vol. 3, no. 1, pp. 1-10, 2014. 
[16] F. Zendeboodi, N. Khorshidian, A. M. Mortazavian, and A. G. Da Cruz, "Probiotic: conceptualization from a new approach," Current Opinion in Food Science, vol. 32, pp. 103-123, 2020.

[17] R. D. Hills, B. A. Pontefract, H. R. Mishcon et al., "Gut microbiome: profound implications for diet and disease," Nutrient, vol. 11, no. 7, pp. 1-40, 2019.

[18] C. J. Hernandez JDG, M. Luna, and S. R. Goldring, "Links between the microbiome and bone," Journal of Bone and Mineral Research: the Official Journal of the American Society for Bone and Mineral Research, vol. 31, no. 9, pp. 1638-1646, 2017.

[19] T. Takimoto, M. Hatanaka, T. Hoshino et al., "Effect of Bacillus subtilis C-3102 on bone mineral density in healthy postmenopausal Japanese women: a randomized, placebocontrolled, double-blind clinical trial," Bioscience of Microbiota, Food and Health, vol. 37, no. 4, pp. 87-96, 2018.

[20] M. N. T. Lambert, C. B. Thybo, S. Lykkeboe et al., "Combined bioavailable isoflavones and probiotics improve bone status and estrogen metabolism in postmenopausal osteopenic women: a randomized controlled trial," The American Journal of Clinical Nutrition, vol. 106, no. 3, p. ajcn153353, 2017.

[21] M. Narva, R. Nevala, T. Poussa, and R. Korpela, "The effect of Lactobacillus helveticus fermented milk on acute changes in calcium metabolism in postmenopausal women," European Journal of Nutrition, vol. 43, no. 2, pp. 61-68, 2004.

[22] I. N. Sergeev, T. Aljutaily, G. Walton, and E. Huarte, "Effects of synbiotic supplement on human gut microbiota, body composition and weight loss in obesity," Nutrients, vol. 12, no. 1, p. 222, 2020.

[23] S. Jafarnejad, K. Djafarian, M. R. Fazeli, M. S. Yekaninejad, A. Rostamian, and S. A. Keshavarz, "Effects of a multispecies probiotic supplement on bone health in osteopenic postmenopausal women: a randomized, double-blind, controlled trial," Journal of the American College of Nutrition, vol. 36, no. 7, pp. 497-506, 2017.

[24] (US) OotSG, One Health and Osteoporosis: A Report of the Surgeon General, Office of the Surgeon General (US), Washington, DC, USA, 2004.

[25] World Health Organization Collaborating Center, Belgium on Behalf of the, Advisors ICoS. Osteoporosis in the Workplace, the Social, Economic and Human Costs of Osteoporosis on Employees, Employers and GovernmentsWorld Health Organization Collaborating Center, Geneva, Switzerland, 2002, https://www.iofbonehealth.org/.

[26] C. S. Colón-Emeric and K. G. Saag, "Osteoporotic fractures in older adults," Best Practice \& Research Clinical Rheumatology, vol. 20, no. 4, pp. 695-706, 2006.

[27] J. P. T. Higgins, D. G. Altman, P. C. Gotzsche et al., "The cochrane collaboration's tool for assessing risk of bias in randomised trials," BMJ, vol. 343, p. d5928, 2011.

[28] R. Mutuş, N. Kocabağli, M. Alp, N. Acar, M. Eren, and Ş. Ş. Gezen, "The effect of dietary probiotic supplementation on tibial bone characteristics and strength in broilers," Poultry Science, vol. 85, no. 9, pp. 1621-1625, 2006.

[29] M. Houshmand, K. Azhar, I. Zulkifli, M. H. Bejo, A. Meimandipour, and A. Kamyab, "Effects of non-antibiotic feed additives on performance, tibial dyschondroplasia incidence and tibia characteristics of broilers fed low-calcium diets," Journal of Animal Physiology and Animal Nutrition, vol. 95, no. 3, pp. 351-358, 2011.

[30] F. F. Yan, A. A. Mohammed, G. R. Murugesan, and H. W. Cheng, "Effects of a dietary synbiotic inclusion on bone health in broilers subjected to cyclic heat stress episodes," Poultry Science, vol. 98, no. 3, pp. 1083-1089, 2019.

[31] F. F. Yan, G. R. Murugesan, and H. W. Cheng, "Effects of probiotic supplementation on performance traits, bone mineralization, cecal microbial composition, cytokines and corticosterone in laying hens," Animal, vol. 13, no. 1, pp. 33-41, 2019.

[32] M. Narva, J. Rissanen, J. Halleen, H. Vapaatalo, K. Väänänen, and R. Korpela, "Effects of bioactive peptide, valyl-prolylproline (VPP), and lactobacillus helveticus fermented milk containing VPP on bone loss in ovariectomized rats," Annals of Nutrition and Metabolism, vol. 51, no. 1, pp. 65-74, 2007.

[33] S. Takasugi, K. Ashida, S. Maruyama, Y. Komaba, T. Kaneko, and T. Yamaji, "A dairy product fermented by lactobacilli cancels the adverse effects of hypochlorhydria induced by a proton pump inhibitor on bone metabolism in growing rats," British Journal of Nutrition, vol. 106, no. 10, pp. 1487-1494, 2011.

[34] K.-S. Shim, T. Kim, H. Ha et al., "Hwangryun-haedok-tang fermented withLactobacillus caseiSuppresses ovariectomyinduced bone loss," Evidence-Based Complementary and Alternative Medicine, vol. 201212 pages, 2012.

[35] S. Takasugi, K. Ashida, S. Maruyama, Y. Matsukiyo, T. Kaneko, and T. Yamaji, "A combination of a dairy product fermented by lactobacilli and galactooligosaccharides shows additive effects on mineral balances in growing rats with hypochlorhydria induced by a proton pump inhibitor," $B i$ ological Trace Element Research, vol. 153, no. 1-3, pp. 309-318, 2013.

[36] K. Parvaneh, M. Ebrahimi, M. R. Sabran et al., "Probiotics (Bifidobacterium longum) increase bone mass density and upregulate sparc and Bmp-2 genes in rats with bone loss resulting from ovariectomy," BioMed Research International, vol. 201510 pages, 2015.

[37] M. Parvaneh, G. Karimi, R. Jamaluddin, A. M. H. Ng, Z. Ibrahim, and S. I. Muhammad, "Lactobacillus helveticus (ATCC 27558) upregulates Runx2 and Bmp2 and modulates bone mineral density in ovariectomy-induced bone loss rats," Clinical Interventions in Aging, vol. 13, pp. 1555-1564, 2018.

[38] M. Bayat, M. H. Dabbaghmanesh, F. Koohpeyma, M. Mahmoodi, N. Montazeri-Najafabady, and M. Bakhshayeshkaram, "The effects of soy milk enriched with lactobacillus casei and omega- 3 on the tibia and L5 vertebra in diabetic rats: a stereological study," Probiotics and Antimicrobial Proteins, vol. 11, no. 4, pp. 1172-1181, 2019.

[39] Y. M. Lee, I. S. Kim, and B. O. Lim, "Black rice (Oryza sativa L.) fermented with lactobacillus casei attenuates osteoclastogenesis and ovariectomy-induced osteoporosis," BioMed Research International, vol. 201916 pages, 2019.

[40] N. Montazeri-Najafabady, Y. Ghasemi, M. H. Dabbaghmanesh, P. Talezadeh, F. Koohpeyma, and A. Gholami, "Supportive role of probiotic strains in protecting rats from ovariectomy-induced cortical bone loss," Probiotics and Antimicrobial Proteins, vol. 11, no. 4, pp. 1145-1154, 2019.

[41] M. Narva, M. Collin, C. Lamberg-Allardt et al., "Effects of long-term intervention with Lactobacillus helveticus-fermented milk on bone mineral density and bone mineral content in growing rats," Annals of Nutrition and Metabolism, vol. 48, no. 4, pp. 228-234, 2004.

[42] S.-S. Chiang and T.-M. Pan, "Antiosteoporotic effects of Lactobacillus-fermented soy skim milk on bone mineral density and the microstructure of femoral bone in ovariectomized mice," Journal of Agricultural and Food Chemistry, vol. 59, no. 14, pp. 7734-7742, 2011. 
[43] L. R. McCabe, R. Irwin, L. Schaefer, and R. A. Britton, "Probiotic use decreases intestinal inflammation and increases bone density in healthy male but not female mice," Journal of Cellular Physiology, vol. 228, no. 8, pp. 1793-1798, 2013.

[44] C. Ohlsson, C. Engdahl, F. Fåk et al., "Probiotics protect mice from ovariectomy-induced cortical bone loss," PLoS One, vol. 9, no. 3, p. e92368, 2014.

[45] J. Zhang, K. J. Motyl, R. Irwin, O. A. MacDougald, R. A. Britton, and L. R. McCabe, "Loss of bone and Wnt10b expression in male type 1 diabetic mice is blocked by the probiotic lactobacillus reuteri," Endocrinology, vol. 156, no. 9, pp. 3169-3182, 2015.

[46] F. L. Collins, N. D. Rios-Arce, J. D. Schepper et al., "Beneficial effects of Lactobacillus reuteri 6475 on bone density in male mice is dependent on lymphocytes," Scientific Reports, vol. 9, no. 1, p. 14708, 2019.

[47] D.-E. Kim, J.-K. Kim, S.-K. Han, S.-E. Jang, M. J. Han, and D.-H. Kim, "Lactobacillus plantarum NK3 and Bifidobacterium longum NK49 alleviate bacterial vaginosis and osteoporosis in mice by suppressing NF- $\kappa$ B-Linked TNF- $\alpha$ expression," Journal of Medicinal Food, vol. 22, no. 10, pp. 1022-1031, 2019.

[48] H. Liu, R. Gu, W. Li et al., "Probiotics protect against tenofovir-induced mandibular bone loss in mice by rescuing mandible-derived mesenchymal stem cell proliferation and osteogenic differentiation," Journal of Oral Rehabilitation, vol. 47, no. S1, pp. 83-90, 2019.

[49] H. Liu, R. Gu, W. Li et al., "Lactobacillus rhamnosus GG attenuates tenofovir disoproxil fumarate-induced bone loss in male mice via gut-microbiota-dependent anti-inflammation," Therapeutic Advances in Chronic Disease, vol. 10, p. $204062231986065,2019$.

[50] L. Lawenius, J. M. Scheffler, K. L. Gustafsson et al., "Pasteurized Akkermansia muciniphila protects from fat mass gain but not from bone loss," American Journal of PhysiologyEndocrinology and Metabolism, vol. 318, no. 4, pp. E480-E491, 2020.

[51] J. Mathey, J. Mardon, N. Fokialakis et al., "Modulation of soy isoflavones bioavailability and subsequent effects on bone health in ovariectomized rats: the case for equol," Osteoporosis International, vol. 18, no. 5, pp. 671-679, 2007.

[52] F. C. Rodrigues, A. S. B. Castro, V. C. Rodrigues et al., "Yacon flour and bifidobacterium longum modulate bone health in rats," Journal of Medicinal Food, vol. 15, no. 7, pp. 664-670, 2012.

[53] S. C. Achi, R. R. Talahalli, and P. M. Halami, "Prophylactic effects of probiotic Bifidobacterium spp. in the resolution of inflammation in arthritic rats," Applied Microbiology and Biotechnology, vol. 103, no. 15, pp. 6287-6296, 2019.

[54] S. Eaimworawuthikul, W. Tunapong, T. Chunchai et al., "Effects of probiotics, prebiotics or synbiotics on jawbone in obese-insulin resistant rats," European Journal of Nutrition, vol. 58, no. 7, pp. 2801-2810, 2019.

[55] Y. L. D. A. S. Levi, R. N. Picchi, E. K. T. Silva et al., "Probiotic administration increases mandibular bone mineral density on rats exposed to cigarette smoke inhalation," Brazilian Dental Journal, vol. 30, no. 6, pp. 634-640, 2019.

[56] R. A. Britton, R. Irwin, D. Quach et al., "Probiotic L. reuteri treatment prevents bone loss in a menopausal ovariectomized mouse model," Journal of Cellular Physiology, vol. 229, no. 11, pp. 1822-1830, 2014.

[57] F. L. Collins, R. Irwin, H. Bierhalter et al., "Lactobacillus reuteri 6475 increases bone density in intact females only under an inflammatory setting," PLoS One, vol. 11, no. 4, p. e0153180, 2016.

[58] J. D. Schepper, R. Irwin, J. Kang et al., "Probiotics in gut-bone signaling," Advances in Experimental Medicine and Biology, vol. 1033, pp. 225-247, 2017.

[59] H. Y. Dar, P. Shukla, P. K. Mishra et al., "Lactobacillus acidophilus inhibits bone loss and increases bone heterogeneity in osteoporotic mice via modulating Treg-Th17 cell balance," Bone Reports, vol. 8, pp. 46-56, 2018.

[60] H. Kimoto-Nira, C. Suzuki, M. Kobayashi, K. Sasaki, J.-i. Kurisaki, and K. Mizumachi, "Anti-ageing effect of a lactococcal strain: analysis using senescence-accelerated mice," British Journal of Nutrition, vol. 98, no. 6, pp. 1178-1186, 2007.

[61] H. Kimoto-Nira, K. Mizumachi, T. Okamoto, K. Sasaki, and J.-I. Kurisaki, "Influence of long-term consumption of a Lactococcus lactis strain on the intestinal immunity and intestinal flora of the senescence-accelerated mouse," British Journal of Nutrition, vol. 102, no. 2, pp. 181-185, 2009.

[62] K. E. Scholz-Ahrens, B. Adolphi, F. Rochat et al., "Effects of probiotics, prebiotics, and synbiotics on mineral metabolism in ovariectomized rats-impact of bacterial mass, intestinal absorptive area and reduction of bone turn-over," NFS Journal, vol. 3, pp. 41-50, 2016.

[63] C. Blanton, "Bone response to dietary co-enrichment with powdered whole grape and probiotics," Nutrients, vol. 10, no. 2, p. 146, 2018.

[64] J. D. Schepper, F. L. Collins, N. D. Rios-Arce et al., "Probiotic lactobacillus reuteri prevents postantibiotic bone loss by reducing intestinal dysbiosis and preventing barrier disruption," Journal of Bone and Mineral Research, vol. 34, no. 4, pp. 681-698, 2019.

[65] S.-S. Chiang, J.-W. Liao, and T.-M. Pan, "Effect of bioactive compounds in lactobacilli-fermented soy skim milk on femoral bone microstructure of aging mice," Journal of the Science of Food and Agriculture, vol. 92, no. 2, pp. 328-335, 2012.

[66] K.-S. Shim, T. Kim, H. Ha et al., "Lactobacillus fermentation enhances the inhibitory effect of Hwangryun-haedok-tang in an ovariectomy-induced bone loss," BMC Complementary and Alternative Medicine, vol. 13, no. 1, p. 106, 2013.

[67] K. E. Scholz-Ahrens, P. Ade, B. Marten et al., "Prebiotics, probiotics, and synbiotics affect mineral absorption, bone mineral content, and bone structure," The Journal of Nutrition, vol. 137, no. 3, pp. 838S-846S, 2007.

[68] A. G. Nilsson, D. Sundh, F. Bäckhed, and M. Lorentzon, "Lactobacillus reuterireduces bone loss in older women with low bone mineral density: a randomized, placebo-controlled, double-blind, clinical trial," Journal of Internal Medicine, vol. 284, no. 3, pp. 307-317, 2018.

[69] M. L. Jones, C. J. Martoni, and S. Prakash, "Oral supplementation with probiotic L. reuteri NCIMB 30242 increases mean circulating 25-hydroxy vitamin D: a post hoc analysis of a randomized controlled trial," The Journal of Clinical Endocrinology \& Metabolism, vol. 98, no. 7, pp. 2944-2951, 2013.

[70] A. Sue and D. S. Shapses, "Bone metabolism in obesity and weight loss," Annual Review of Nutrition, vol. 32, pp. 287-309, 2012.

[71] H.-S. Ejtahed, P. Angoorani, A.-R. Soroush et al., "Probiotics supplementation for the obesity management; A systematic review of animal studies and clinical trials," Journal of Functional Foods, vol. 52, pp. 228-242, 2019.

[72] H.-S. Ejtahed, P. Angoorani, S. Hasani-Ranjbar et al., "Adaptation of human gut microbiota to bariatric surgeries in 
morbidly obese patients: a systematic review," Microbial Pathogenesis, vol. 116, pp. 13-21, 2018.

[73] D. Jones, L. H. Glimcher, and A. O. Aliprantis, "Osteoimmunology at the nexus of arthritis, osteoporosis, cancer, and infection," Journal of Clinical Investigation, vol. 121, no. 7, pp. 2534-2542, 2011.

[74] A. Koh, F. De Vadder, P. Kovatcheva-Datchary, and F. Bäckhed, "From dietary fiber to host physiology: shortchain fatty acids as key bacterial metabolites," Cell, vol. 165, no. 6, pp. 1332-1345, 2016.

[75] S. Lucas, Y. Omata, J. Hofmann et al., "Short-chain fatty acids regulate systemic bone mass and protect from pathological bone loss," Nature Communications, vol. 9, no. 1, p. 55, 2018.

[76] H.-S. Ejtahed, A.-R. Soroush, P. Angoorani, B. Larijani, and S. Hasani-Ranjbar, "Gut microbiota as a target in the Pathogenesis of metabolic disorders: a new approach to novel therapeutic agents," Hormone and Metabolic Research, vol. 48, no. 6, pp. 349-358, 2016.

[77] H. S. Ejtahed, S. Hasani-Ranjbar, and B. Larijani, "Human microbiome as an approach to personalized medicine," Alternative Therapies in Health and Medicine, vol. 23, no. 6, pp. 8-9, 2017.

[78] J. Yan, A. Takakura, K. Zandi-Nejad, and J. F. Charles, "Mechanisms of gut microbiota-mediated bone remodeling," Gut Microbes, vol. 9, no. 1, pp. 84-92, 2018.

[79] X. Li, X. Li, Q. Shang et al., "Fecal microbiota transplantation (FMT) could reverse the severity of experimental necrotizing enterocolitis (NEC) via oxidative stress modulation," Free Radical Biology and Medicine, vol. 108, pp. 32-43, 2017.

[80] H. Kalyanaraman, N. Schall, and R. B. Pilz, "Nitric oxide and cyclic GMP functions in bone," Nitric Oxide, vol. 76, pp. $62-70,2018$.

[81] J. D. Schepper, R. Irwin, J. Kang et al., "Probiotics in gut-bone signaling," Advances in Experimental Medicine and Biology, vol. 1033, pp. 225-247, 2017.

[82] P. Hemarajata and J. Versalovic, "Effects of probiotics on gut microbiota: mechanisms of intestinal immunomodulation and neuromodulation," Therapeutic Advances in Gastroenterology, vol. 6, no. 1, pp. 39-51, 2013.

[83] S. Cheng, X. Qi, M. Ma et al., "Assessing the relationship between gut microbiota and bone mineral density," Frontiers in Genetics, vol. 11, no. 6, 2020.

[84] J. Yan, J. W. Herzog, K Tsang et al., "Gut microbiota induce IGF-1 and promote bone formation and growth," Proceedings of the National Academy of Sciences of the United States of America, vol. 113, no. 47, p. E7554, 2016.

[85] V. Locatelli and V. E. Bianchi, "Effect of GH/IGF-1 on bone metabolism and osteoporsosis," International Journal of Endocrinology, vol. 201425 pages, 2014.

[86] S. Li, Y. Mao, F. Zhou, H. Yang, Q. Shi, and B. Meng, "Gut microbiome and osteoporosis," Bone \& Joint Research, vol. 9, p. 8,2020 .

[87] M. Almeida, M. R. Laurent, V. Dubois et al., "Estrogens and androgens in skeletal physiology and pathophysiology," Physiological Reviews, vol. 97, no. 1, pp. 135-187, 2017.

[88] M. L. Hao, G. Y. Wang, X. Q. Zuo, C. J. Qu, B. C. Yao, and D. L. Wang, "Gut microbiota: an overlooked factor that plays a significant role in osteoporosis," The Journal of International Medical Research, vol. 47, pp. 4095-4103, 2019. 